\title{
A Nature-inspired Hierarchical Branching Structure Pressure Sensor for Medical Wearables
}

Huang Guoliang ( $\nabla$ tshgl@mail.tsinghua.edu.cn )

Tsinghua University

Yang Han

Tsinghua University https://orcid.org/0000-0002-4475-4803

Fu Rongxin

Tsinghua University

Shan Xiaohui

Tsinghua University

Wang Ruliang

Tsinghua University

Lin Xue

Tsinghua University

Su Ya

Tsinghua University

Jin Xiangyu

Tsinghua University

Du Wenli

Tsinghua University https://orcid.org/0000-0002-4518-4558

Lv Wenqi

Tsinghua University

\section{Article}

Keywords: nature-inspired, hierarchical branching network, flexible pressure sensor, graphene, medical wearables

Posted Date: April 23rd, 2021

DOl: https://doi.org/10.21203/rs.3.rs-421121/v1

License: (9) (1) This work is licensed under a Creative Commons Attribution 4.0 International License. Read Full License 


\section{A Nature-inspired Hierarchical Branching Structure Pressure Sensor for Medical Wearables}

Both ecosystems and biological systems in nature have evolved with unique hierarchical branching networks to maximize the efficiency and range of material transmission and adaptability to their external environment. Here we emulate this hierarchical branching (HB) network using a multilayer superposition approach and apply it in the design of flexible pressure sensors for medical wearables. Based on the HB structure, simulation results demonstrate that the deformation of microstructures is efficiently scheduled. Experiments show that the sensitivity of the HB sensor exhibits almost 34- and 55-fold improvements over the medium-pressure and high-pressure ranges, respectively, compared with that of a pressure sensor with a traditional monolayer structure. Successful monitoring of the diverse stimuli from humans demonstrates the considerable potential of the HB sensor in medical wearables. Additionally, the small and thin nature of the sensor enables it to quantify medical diagnostic processes, such as intelligent traditional Chinese medicine pulse diagnosis.

KEYWORDS: nature-inspired, hierarchical branching network, flexible pressure sensor, graphene, medical wearables.

Over millions of years of evolution and selection, both ecosystems and biological systems in nature have skillfully evolved excellent structures from a fairly limited choice of compounds. ${ }^{1}$ 
Such biological structures offer a spectrum of feasible pathways for the design of functional materials towards unprecedented functionalities and properties that are favorable for practical applications in engineering and medical fields. ${ }^{2,3}$ For example, to maximum the efficiency of material transmission with minimum energy consumption, both natural world (e.g., the leaf venations of trees) and human life (e.g., the windpipe network in lungs, the blood vessel network in human bodies, the water supplies of a city) has evolved similar hierarchical branching networks, which is initiated with the largest pores and extended by multi-scale nodes with increasing numbers but decreasing diameters. ${ }^{4-6}$ The principle of this optimized hierarchical branching network is commonly referred to as Murray's law, which provides an optimized design for porous materials to maximize the transfer efficiency. ${ }^{7,8}$ As a multi-functional interface to interact with the physical world, human skin has evolved excellent somatosensory system, ${ }^{9}$ which put forward higher requirements for electronic skins. To meet the growing demand of biomedical application, nextgeneration sensors aim to improve the key performance parameters, such as sensitivity and pressure detection range. ${ }^{10}$ Analogous to the universality and principle of such hierarchical branching networks in nature world and human life, such network may be a feasible pathway to optimized the performance of electronic skins in biomedical engineering.

Recent developments in wearable flexible electronic devices for human health monitoring and human-machine interactions have attracted much attention. ${ }^{11-14}$ Flexible pressure sensors, which mimic the perceptive function of the human dermis, exhibit great potential in health monitoring devices ${ }^{15,16}$, biomimetic prostheses ${ }^{17}$ and soft robots. ${ }^{18}$ Generally, the most common sensing mechanism of pressure sensors are mainly classified as resistive, capacitive, piezoelectric and triboelectric types. ${ }^{10}$ Among these types, piezoresistive sensors have been of particular interests due to their simple structure and readout mechanism. In general, a microstructured elastic film 
integrated with sensitive nanomaterials is considered the most popular flexible substrate for piezoresistive pressure sensors. ${ }^{19,20}$ Mainstream research efforts have been devoted to introducing various homogeneous and unitary microstructures (e.g., hemispheres, ${ }^{21,22}$ pyramids, ${ }^{23,24}$ pillars ${ }^{25}$ ) into pressure sensor active layers for improved performance. Under external applied pressure, all the microstructures deform together and contribute to a significant change in the contact resistance. The high sensitivity of these sensors, however, is only valid in limited pressure regions because of the deformation saturation resulting from the elastic modulus. To address these limitations, several geometric microengineering strategies have been employed, including the use of hierarchical structures, ${ }^{26}$ hollow structures,${ }^{27,28}$ novel geometric characteristics (e.g., Gaussian distribution rough surface, ${ }^{29,30}$ plant leaves, ${ }^{31}$ ), gradient conductivity and stiffness, ${ }^{32}$ etc. However, new strategies to achieve a trade-off among the sensitivity, linearity range, easy of fabrication, active layer uniformity, shape and size versatility and tunability, and scalability of both the device and fabrication process for practical applications remain to be explored. ${ }^{10}$

Here, we demonstrate a nature-inspired flexible pressure sensor based on a hierarchical branching structure. The HB structure is realized using a simple multilayer superposition approach, which is tunable and scalable. In this $\mathrm{HB}$ structure design, two microstructured polydimethylsiloxane (PDMS) films serve as the elastic HB structure foundation, and graphene serves as the sensing material. We demonstrate that such HB structure can realize the branch transmission of pressure. Given this advantage, the deformation of microstructures is efficiently scheduled, which will effectively optimize the sensitivity and pressure detection range of pressure sensors. Experimental results showed that the pressure sensor with HB structure exhibits almost 34- and 55-fold improvements in sensitivity over the medium-pressure and high-pressure ranges $(>10 \mathrm{kPa})$, respectively, compared with that of a pressure sensor with a traditional monolayer 
structure. When compared with the previously reported pressure sensors based on multi-layer or hierarchical structure, the proposed HB structure has a more significant effect on optimizing the sensitivity of the sensor. The HB graphene sensor has been validated in healthcare applications and artificial sensory feedback solutions. In addition, the small and thin nature of the sensor enables its application in quantifying medical diagnostic processes, such as intelligent traditional Chinese medicine (TCM) pulse diagnosis. The physician's manipulation and the patient's pulse signal should be recorded simultaneously during this process, where both high sensitivity and broad dynamic response range are required.

\section{RESULTS AND DISCUSSION}

(a)

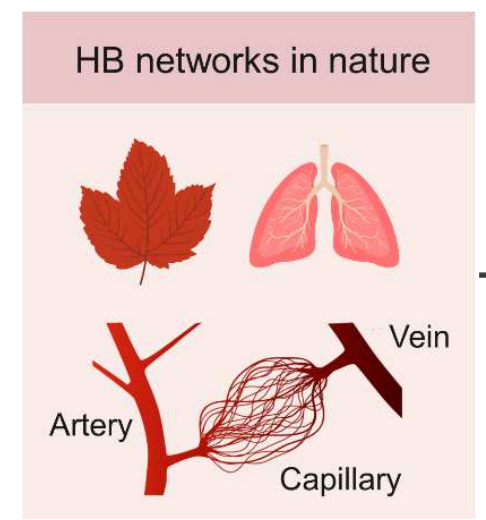

(b)

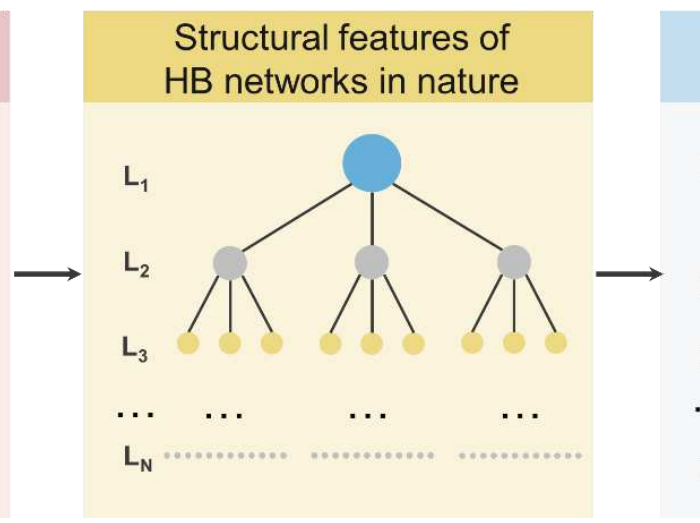

(c)

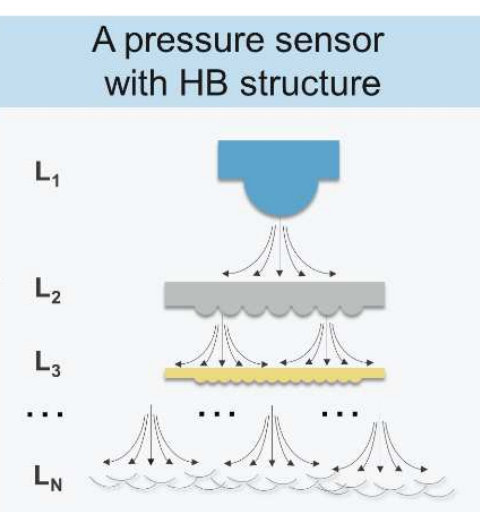

Figure 1. Hierarchical branching networks. (a) Material transport HB networks in nature. (b) Structural features of HB networks in nature. (c) Concept of the nature-inspired HB structure pressure sensor.

Nature-inspired hierarchical branching networks. To maximum the efficiency of material transmission with minimum energy consumption, both natural world and human life has evolved similar hierarchical branching networks. ${ }^{4-6}$ For example, the blood vessels in human bodies and the windpipe in lungs represents as a branching network to maximize the efficiency of material 
transport (Figure 1a) ${ }^{7,8}$ Similarly, plants possess leaf veins to improve the rate of photosynthesis for energy conversion (Figure 1a). ${ }^{2,6}$ In the general case, the structure features of the branching network in nature can be simplified as a topological structure, as shown in Figure $1 b .{ }^{4}$ The topological structure contains $\mathrm{N}$ branches from the aorta (level 0) to the capillaries (level $\mathrm{N}$ ). Inspired by the hierarchical branching networks in nature world and human life, we apply it in the design of flexible pressure sensors to realize the branch transmission of pressure and efficiently schedule the deformation of microstructures.

(a)

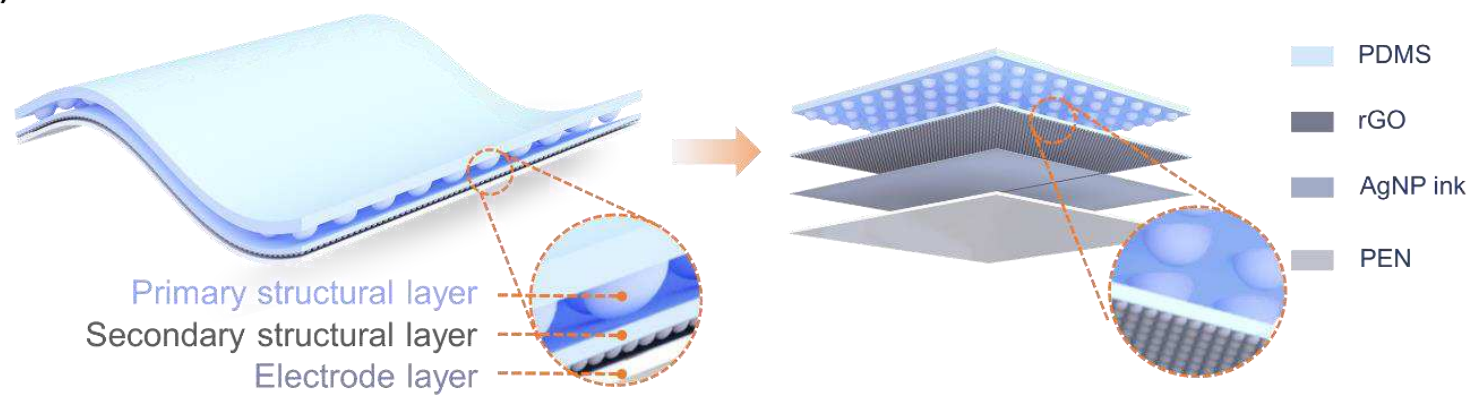

(b)
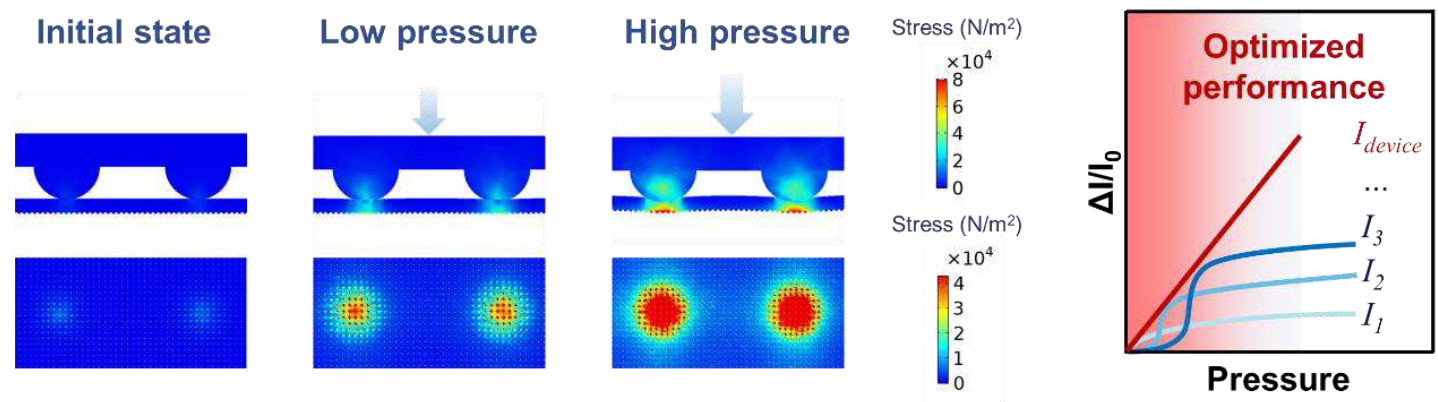

(c)
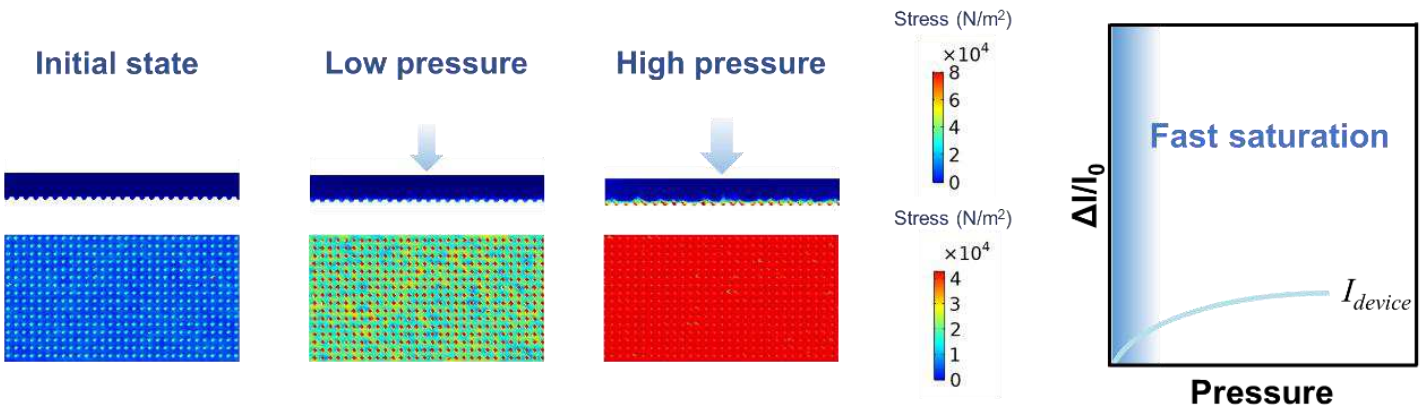
Figure 2. Schematics of the HB pressure sensor. (a) Schematic diagram of the pressure sensor which contains two pressure-sensitive microdome-structured layers and a side-by-side electrodes layer. (b) Deformation and surface von Mises stress of pressure sensors with HB structure. This illustration shows the operating principle of the HB structure. Under applied pressure, the number of contacting microstructures increases, which contributes to improving the sensitivity and linearity range. (c) Deformation and surface von Mises stress of pressure sensors with monolayer regular periodic microstructures.

Structure of the HB pressure sensor. Figure 2a schematically illustrates the HB structure of the pressure sensor. The HB sensor consists of two pressure-sensitive microdome-structured layers with multi-scale diameters and one printed side-by-side electrode layer. The secondary layer acts as a conductive bridge between the separated coplanar electrodes at the bottom. Figure $2 \mathrm{~b}$ and $2 \mathrm{c}$ illustrates the different sensing mechanisms between the HB and monolayer pressure sensors. The working principle of this $\mathrm{HB}$ structure (gradually mobilizing the microstructure deformation) is shown in Figure $2 \mathrm{~b}$. With increasing pressure, the deformation of the structures in the primary layer will form pressure diffusion effect in the secondary layer and contribute to two kinds of effects at the same time. One is the increasing number of deformable microstructures, and the other is the increasing contact area of the individual microstructures. Although the microstructures located at the center of the primary structure saturate within the low-pressure range, it will be compensated by the microstructures far away from the center. Finally, as explained in Figure 2b, the HB sensor shows enhanced sensitivity and linearity range, attributed to the gradual activation of current pathways radiating out from the center. However, for the traditional pressure sensors with monolayer regular periodic microstructures, all the microstructures deform together and saturate within the low-pressure range (Figure 2c). Generally, the high sensitivity of these sensors 
is valid in limited pressure regions. ${ }^{33}$

(a)
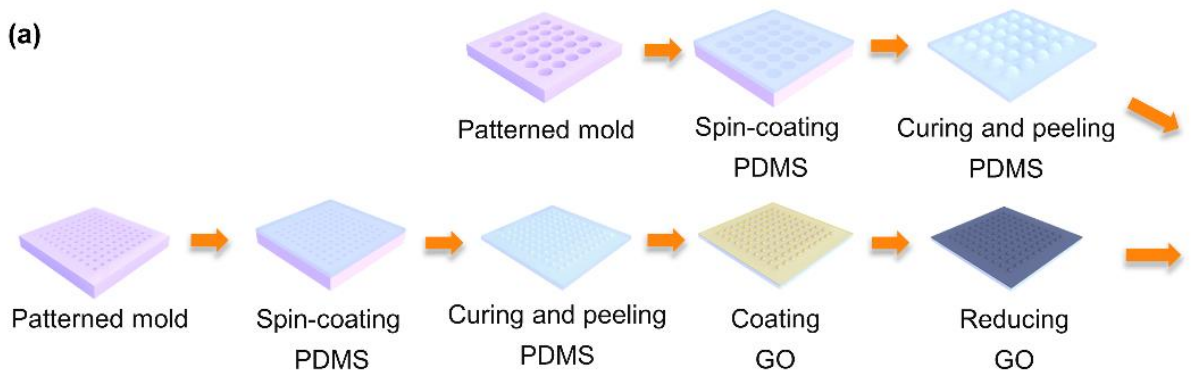

GO

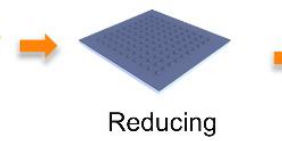

GO
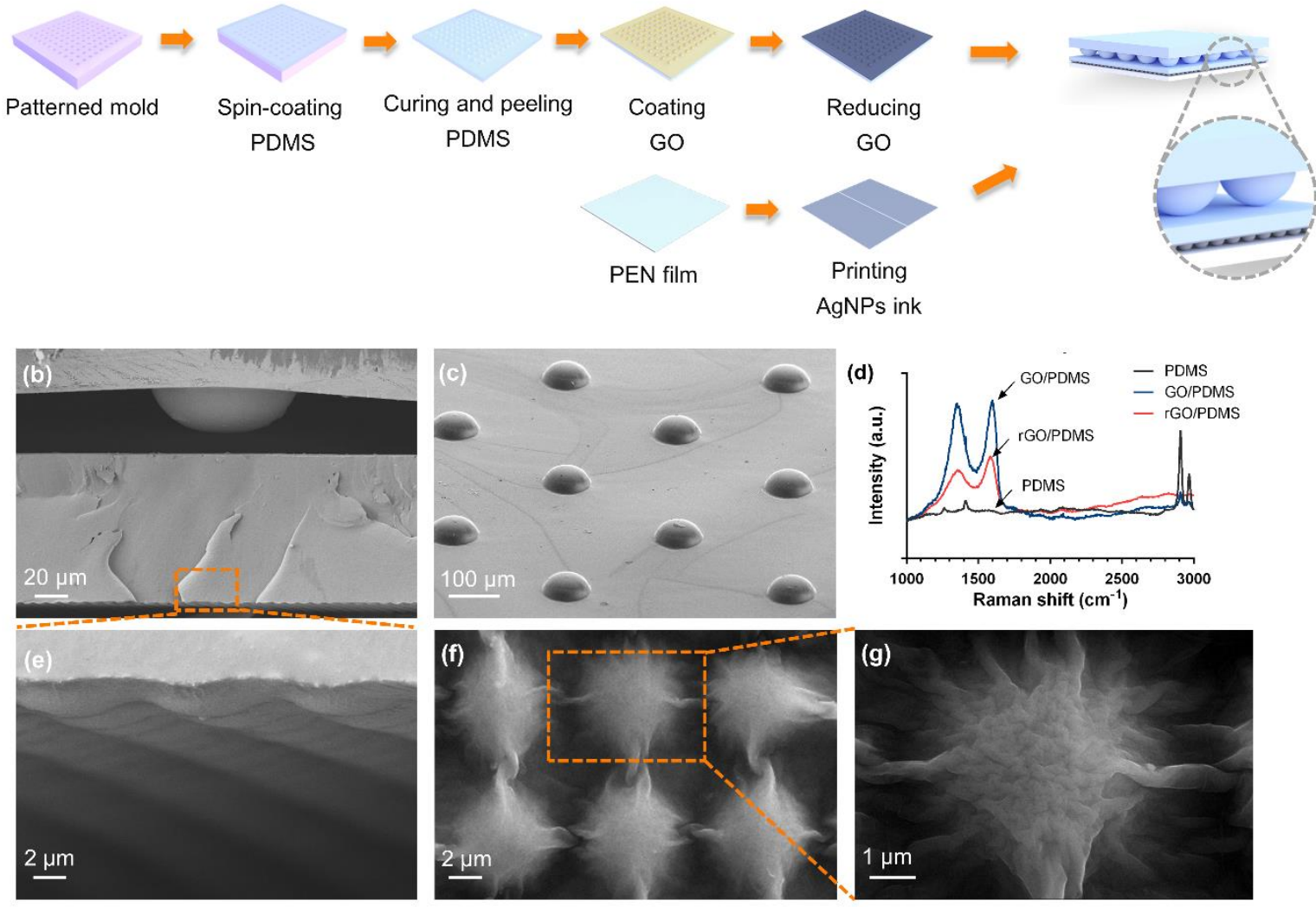

Figure 3. Fabrication and characterization of the prepared HB graphene sensors. (a)

Schematic illustration of the overall fabrication process. (b) Cross-sectional SEM image of the primary layer and secondary layer. (c) Tilted SEM image of the micro-dome array of the PDMS primary layer. (d) Raman spectra of the PDMS, PDMS/GO, and PDMS/rGO. (e) Corresponding magnified SEM image of the secondary layer in (b). (f) Plane magnified SEM image and (g) corresponding magnified SEM image of the PDMS/rGO secondary structural layer.

Fabrication of the HB pressure sensor. The overall fabrication procedure is illustrated in Figure 3a. First, the PDMS precursor was cast on a microdome-patterned mold with different radius and 
cured to obtain micropatterned flexible substrates. Subsequently, graphene oxide (GO) was spraycoated on the structural surface of the flexible substrate with smaller microstructures, which served as the secondary layer, followed by the reduction of GO to reduced graphene oxide (rGO) at a high temperature. The side-by-side electrodes were fabricated on a flexible substrate using inkjetprinting Ag nanoparticle (AgNP) ink. Figure S6 shows the optical micrograph and photographs of the electrodes and the electronic circuit printer. Finally, the two micro-dome-patterned substrates were stacked together on the electrodes, with the PDMS substrate with larger hemispheres serving as the upper layer and the rGO-coated PDMS substrate with smaller hemispheres serving as the middle layer.

Figure $3 \mathrm{~b}$ shows a cross-sectional scanning electron microscopy (SEM) image of the HB structure. The larger microdomes in the primary layer were $100 \mu \mathrm{m}$ in diameter and $300 \mu \mathrm{m}$ in separation distance (Figure 3c), while the smaller microdomes in the secondary layer were $6 \mu \mathrm{m}$ in diameter and $8 \mu \mathrm{m}$ in center distance (Figure 3e). The coverage and morphology of rGO on the overall PDMS film are shown in Figure $3 \mathrm{f}$ and $2 \mathrm{~g}$. The SEM image of the PDMS/rGO film was obtained without any deposition of a conducting material, indicating that the conductive rGO layer fully covered the PDMS film. The SEM images of the PDMS/rGO film with the same structural parameters as the above primary structural layer are shown in Figure S7, which were used as the comparison samples in the subsequent sensitivity comparison experiment. Moreover, due to the expansion of $\mathrm{CO}_{2}$ that evolved into the interstices between the graphene sheets during rapid heating, some cracks were observed in the rGO layer (Figure S7c, d) ${ }^{34}$ which may contribute to the resistance variation. Optical micrographs and photographs of the micropatterned PDMS, PDMS/GO, and PDMS/rGO substrates are shown in Figure S5.

The Raman spectra of PDMS, PDMS/GO, and PDMS/rGO in Figure 3d reveal the quality and 
number of the rGO layers. For the micropatterned PDMS film, there were four characteristic peaks around $1264,1411,2908$, and $2965 \mathrm{~cm}^{-1}$, corresponding to the symmetric bend, asymmetric bend, symmetric stretch, and asymmetric stretch of $\mathrm{CH}_{3}$, respectively. ${ }^{35}$ Both PDMS/GO and PDMS/rGO spectra showed the presence of the D and G bands. For PDMS/GO, the G band was located around $1600 \mathrm{~cm}^{-1}$, while for PDMS/rGO, the G band shifted to $1586 \mathrm{~cm}^{-1}$ due to the reduction of the oxygen functional groups, indicating the successful reduction of GO during the high-temperature treatment. ${ }^{36}$ In addition, the D bands of PDMS/GO and PDMS/rGO can be identified at approximately $1350 \mathrm{~cm}^{-1}$ and $1347 \mathrm{~cm}^{-1}$, respectively, revealing the defects or heteroatom doping in the graphene layer. ${ }^{37}$ Overall, the above results indicate that rGO was successfully reduced and fully covered the micropatterned PDMS film. 
(a)

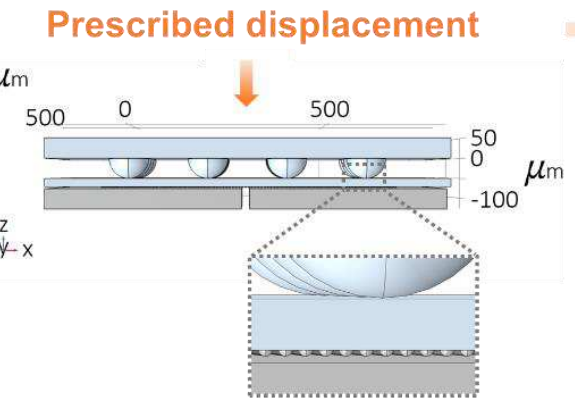

(b)
Prescribed displacement

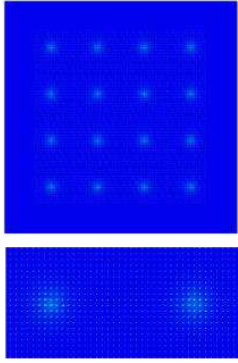

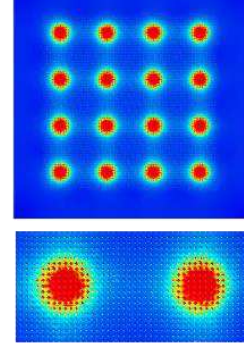

Von Mises Stress $\left(\mathrm{N} / \mathrm{m}^{2}\right)$

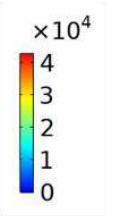

(c)

Low pressure

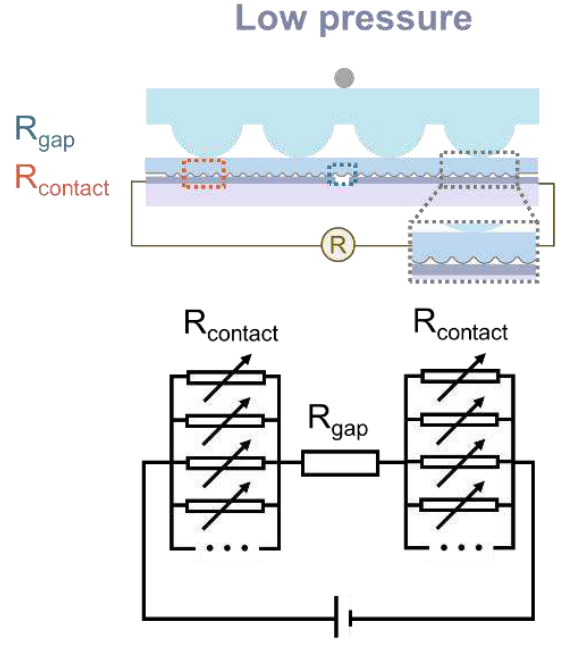

High pressure

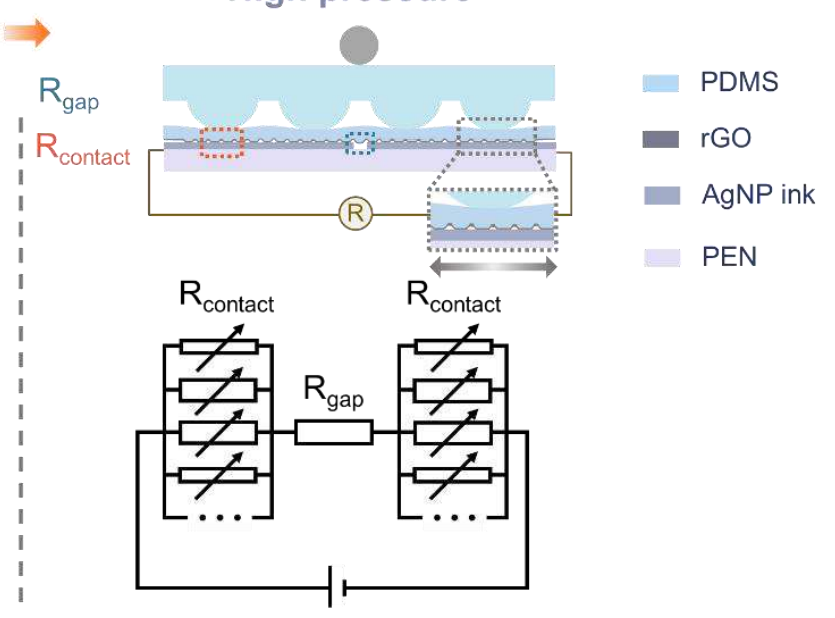

$$
\mathrm{R}_{\text {device }}=2 \mathrm{R}_{\text {single }}+\mathrm{R}_{\text {gap }}=\frac{2}{\mathrm{n} \cdot \frac{1}{\mathrm{R}_{\text {contact }}}}+\mathrm{R}_{\text {gap }}
$$

Figure 4. Working mechanism of the HB pressure sensor. (a) 3D model in the finite element analysis (FEA) software corresponding to the schematic diagram of the pressure sensor in Figure 2a. (b) Simulation results of the pressure distribution and deformation of the microstructure in the secondary structural layer as the prescribed displacement increases. (d) Cross-sectional diagrams of the HB graphene sensor and the corresponding circuit diagrams under low pressure and high pressure.

Working mechanism of the HB pressure sensor. According to the circuit model discussed in 
Section S2 (Supporting Information), there are two key factors that affect the performance of a pressure sensor: the contact area (A) variation under different pressures and the number of microstructures in contact with the electrode (n). For the pressure sensor with a monolayer periodic microarray structure, the device resistance variation only relates to A, while the number of variable contact resistors (n) is fixed (Figure S2b). With increasing applied pressure, all the elastic microstructures tended to saturate, resulting in a significant decrease in sensitivity and a limited linearity range. Based on the analysis of the above mathematical model, this problem can be addressed in two ways. One is solving the deformation saturation, while the other is introducing new microstructures to participate in the deformation, which is the design principle in this work.

To prove whether the HB structure proposed in this work follows the above deformation process, finite element analysis (FEA) was carried out, assuming all the spherical caps were identical. Figure 4a shows the 3D model built in the software, the structural parameters of which are listed in Table S1. As illustrated in Figure 4b, when the prescribed displacement increases, the microstructures in the secondary layer gradually deformed radiating out from the center of the primary structure, compensating for the deformation saturation of the original contacted microstructure, which coincides with our hypothesis. Cross-sectional diagrams of the structural section deformation and corresponding circuit diagrams are shown in Figure 4c. Corresponding to the physical deformation process, with increasing applied pressure, $\mathrm{R}_{\mathrm{cl}}$ of the microstructures near the axis of the primary structure decreases first, while $R_{c 1}$ of the microstructures away from the axis of the primary structure decreases subsequently. This process effectively solves the problem of sensitivity reduction caused by the simultaneous saturation of microstructures. Interestingly, the working mechanism of this HB structure is similar to that of irregular microhump structures, which was studied by Wang et al. ${ }^{30}$ 
(a)

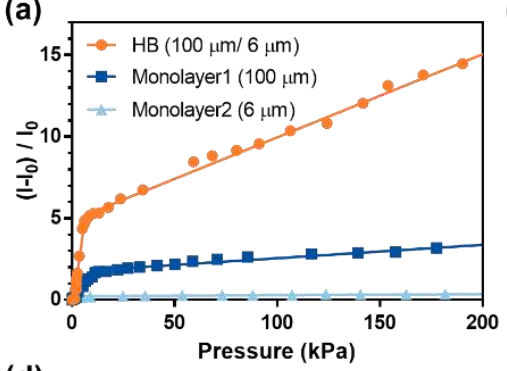

(d)

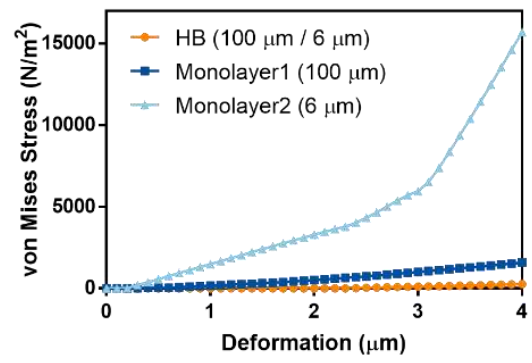

(g)

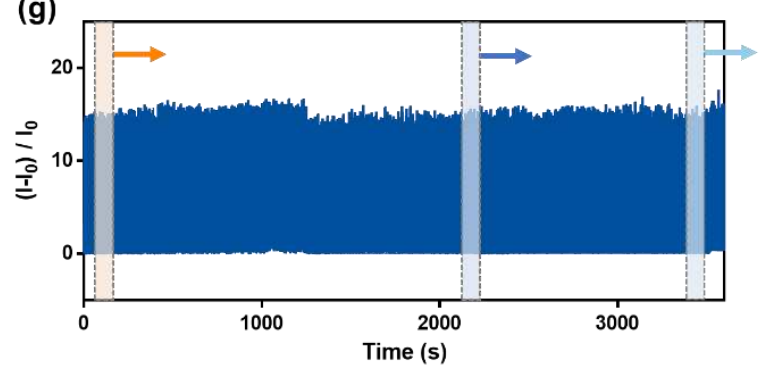

(b)

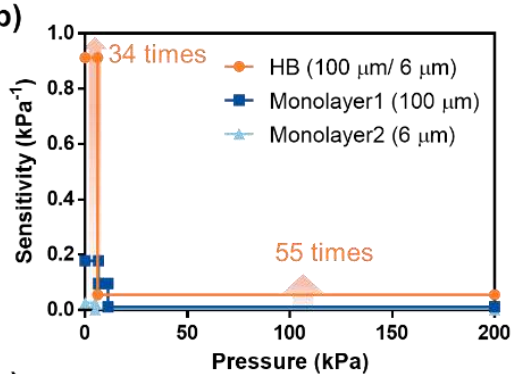

(e)

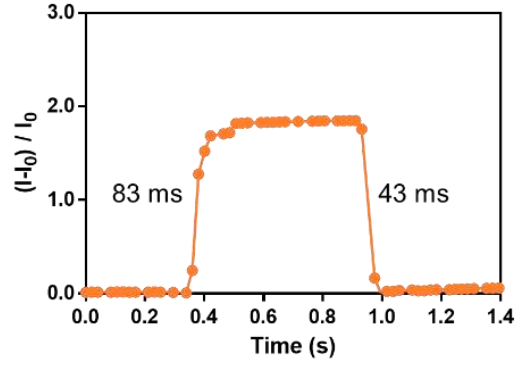

(h) 20

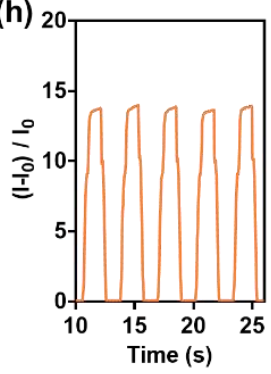

(c)

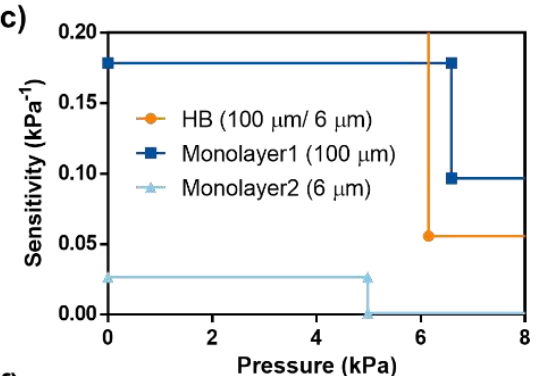

(f)

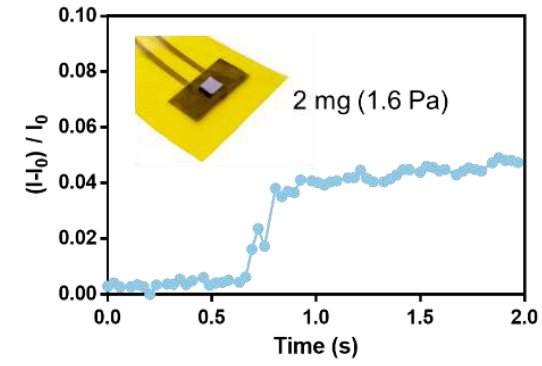

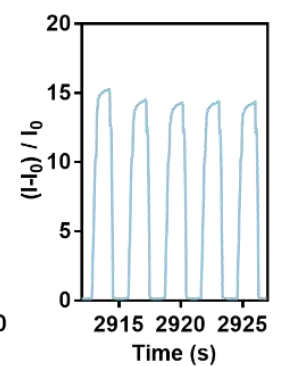

Figure 5. Electromechanical properties of the HB pressure sensor. (a) Relative current variation versus the pressure on the pressure sensors. (b) Performance comparison and (c) the corresponding magnified graph of the three pressure sensors. (d) von Mises stress versus the prescribed displacement for the three pressure sensors. (e) Response and recovery time of the HB graphene sensor with a load of $1.25 \mathrm{kPa}$. (f) Electrical response of the $\mathrm{HB}$ graphene sensor to the subtle pressure of 1.6 Pa. (g) Relative change in resistance under 1000 cycles of loading pressure of $105 \mathrm{kPa}$. The insets show enlarged views of three time periods. (h) The corresponding magnified graph of the dashed box in $(\mathrm{g})$.

Electromechanical properties of the HB pressure sensor. To characterize the effect of the HB structure on the sensor sensitivity and linearity range, the relative current change $\left(\Delta I / I_{0}\right)$ was 
measured as a function of the applied pressure (Figure 5a). The structural parameters of the pressure sensor with the HB structure and two different monolayer periodic structures are listed in Table S1. The sensitivity $S$ is expressed as follows:

$$
\mathrm{S}=(\Delta \mathrm{I} / \mathrm{I}) / \Delta \mathrm{P}
$$

where $\mathrm{I}_{0}$ is the initial current and $\Delta \mathrm{I}\left(=\mathrm{I}-\mathrm{I}_{0}\right)$ is the variation in the current under an external applied pressure $(\mathrm{P})$. As depicted in Figure $5 \mathrm{~b}$ and $\mathrm{c}$, the HB sensor exhibited a sensitivity of $0.91 \mathrm{kPa}^{-1}$ over a linearity range of $0-6.15 \mathrm{kPa}$. Compared to the pressure sensor with a traditional monolayer microstructure (with microstructure diameter $6 \mu \mathrm{m}$ ), the HB sensor exhibits almost 34- and 55fold improvements in sensitivity over medium-pressure $(<10 \mathrm{kPa})$ and high-pressure $(>10 \mathrm{kPa})$ ranges, respectively. When compared with the previously reported pressure sensors based on multilayer or hierarchical structure, the proposed HB structure has a more significant effect on optimizing the sensitivity of the sensor. ${ }^{26,38}$ This result can be attributed to the effective scheduling of microstructural deformation by the branching structure. Theoretically, the increased device thickness by this hierarchical structure might reduce the performance. ${ }^{33}$ However, the above result demonstrated that the enhanced contact variation can make up for the negative effect of thickness and significantly enhance the performance. The optimized performance of the HB graphene sensor is in good agreement with the simulated results (Figure S3). In addition, the comparison of the von Mises stress versus deformation for the HB structure and monolayer structure shown in Figure 5d and Figure S4 indicates that the external stress required for the $\mathrm{HB}$ graphene sensor at a certain compression distance is lower than that for the monolayer structural sensor, which illustrates the higher sensitivity of the HB graphene sensor. Furthermore, to investigate the relation between the microdome sizes of primary and secondary layer on sensors performance, the performance of sensors with different size parameters was compared and analyzed through FEA simulation 
(Supporting Information Section S1).

The response and recovery times of the HB graphene sensor were evaluated by loading a weight equivalent to a pressure of $1.25 \mathrm{kPa}$ (corresponding to the pressure of a gentle finger touch). As shown in Figure 5e, the response and recovery times were $83 \mathrm{~ms}$ and $43 \mathrm{~ms}$ respectively, which is comparable to that of human skin $(\sim 30-50 \mathrm{~ms}) .{ }^{39}$ Furthermore, the response time could be further optimized by reducing the thickness of the elastic films, which will reduce the visco-elastic creep. ${ }^{33}$ Subsequently, we characterized the minimum pressure detection limit. Figure $5 \mathrm{f}$ shows that the pressure sensor can immediately respond to a subtle pressure of 1.6 Pa. This pressure corresponds to a mass of $2 \mathrm{mg}$ over an area of $12.25 \mathrm{~mm}^{2}$ (a stainless-steel weight with a side length of 3.5 $\mathrm{mm})$. Such a low detection limit is helpful for the accurate detection of weak physiological signals, such as human pulse waves. With repetitive cycles under a large pressure of $105 \mathrm{kPa}$ for a period of $3 \mathrm{~s}$, the sensor exhibited great stability and durability for up to 1000 cycles (Figure 5g, h). Based on the above performance, the HB sensor is considered suitable for healthcare applications and medical assessments. 
(a)

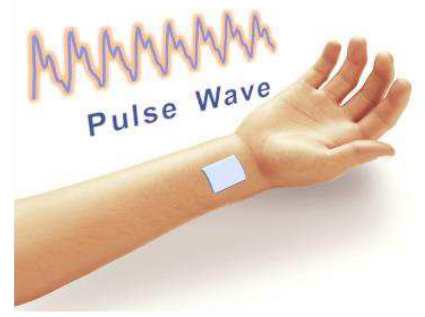

(d)

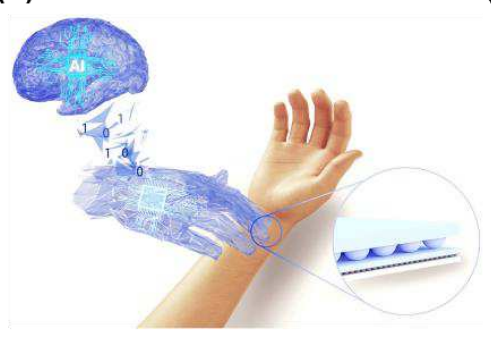

(b)

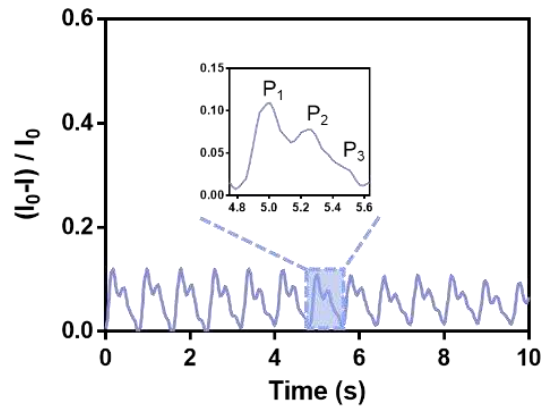

(e)

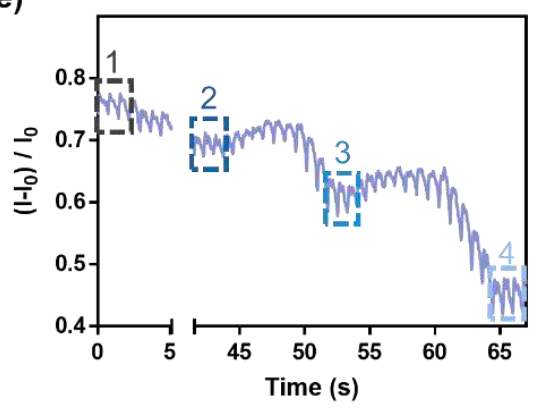

(c)

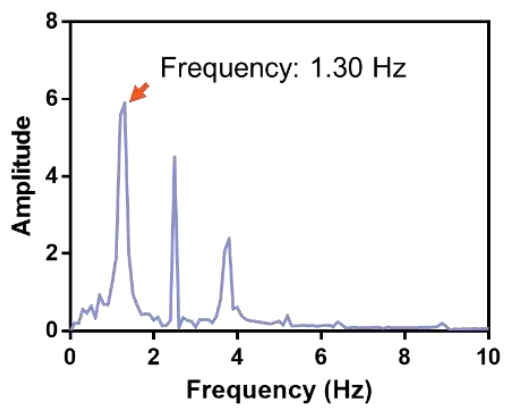

(f)

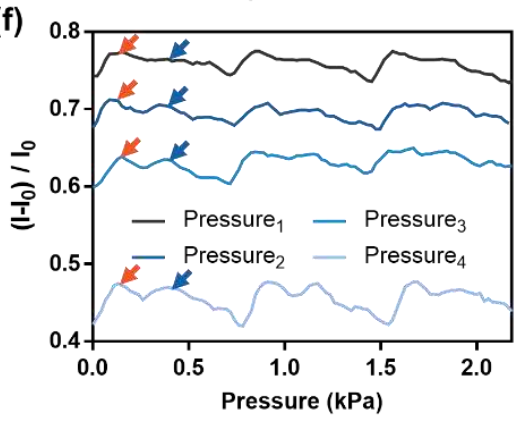

Figure 6. Applications of the HB sensor for pulse monitoring and intelligent pulse diagnosis.

(a) Pulse waveform monitoring schematic showing the HB sensor attached to the radial artery of the wrist. (b) Recorded pulse waveforms; inset shows the detailed pulse waves. (c) FFT results of the recorded pulse waveforms. (d) Schematic of intelligent pulse diagnosis. (e) Recorded pulse waveforms under dynamic pulse-taking pressure. (f) Corresponding mapping results among the waves under four different pulse-taking pressures.

Application examples. In this section, we discuss the potential of the HB sensor in healthcare applications, including real-time radial pulse measurements, breath recording, and electro-tactile feedback for intelligent prosthesis and joint motion control of manipulators. In addition, the small and thin nature of the sensor enables it to be applied in quantifying medical diagnostic processes, such as intelligent pulse diagnosis.

Accurate monitoring of pulse signals plays an important role in cardiovascular health assessments. Derived from the cardiovascular activity, the radial artery pressure wave comprises 
three distinguishable peaks: the systolic peak $\left(\mathrm{P}_{1}\right)$, point of inflection $\left(\mathrm{P}_{2}\right)$, and dicrotic wave $\left(\mathrm{P}_{3}\right)$. Valuable information on cardiovascular health, such as heart rate, vascular aging, and stiffness can be obtained via qualitative analysis of radial artery pressure waves. For example, arterial stiffness can be evaluated using the radial augmentation index $\left(\mathrm{AI}_{\mathrm{r}}=\mathrm{P}_{2} / \mathrm{P}_{1}\right)$, diastolic augmentation index $\left(\mathrm{DAI}_{\mathrm{r}}=\mathrm{P}_{3} / \mathrm{P}_{1}\right)$, and digital volume pulse volume pulse time $\left(\Delta \mathrm{T}_{\mathrm{DVP}}=\mathrm{t}_{\mathrm{p} 2}-\mathrm{t}_{\mathrm{p} 1}\right)$, where $\mathrm{t}_{\mathrm{p} 1}$ and $\mathrm{t}_{\mathrm{p} 2}$ are the occurrence times of the first $\left(\mathrm{P}_{1}\right)$ and second $\left(\mathrm{P}_{2}\right)$ peaks respectively. ${ }^{40}$ The inset in Figure 6a shows an HB sensor affixed to the radial artery of the wrist of a volunteer (24-year-old male) in a seated position. The sensor self-adhered to the skin via silica gel to achieve tight adhesion and conformal contact (Figure S8), which can avoid waveform distortion introduced by the extra holding force. ${ }^{13}$ As shown in Figure 6b, three distinguishable peaks of a typical artery pulse wave can be clearly and steadily distinguished. The calculated average $\mathrm{AI}_{\mathrm{r}}, \mathrm{DAI} \mathrm{I}_{\mathrm{r}}$, and $\Delta \mathrm{T}_{\mathrm{DVP}}$ values are $0.68,0.25$, and $0.14 \mathrm{~s}$, respectively. Notably, the value of $\mathrm{AI}_{\mathrm{r}}$ is slightly higher than the normal health values for people in their mid-20s, ${ }^{40}$ which correlates with the slightly higher diastolic blood pressure of the volunteer $(130 / 90 \mathrm{mmHg}){ }^{41}$ In addition, the corresponding fast Fourier transformation (FFT) results shown in Figure 6c indicated that the harmonic components of the collected pulse wave were mainly distributed in the range of $0-10 \mathrm{~Hz}$, corresponding to that of healthy adults, ${ }^{42}$ which proved that the response time of the HB sensor meets the requirement of pulse measurement. The calculated heart rate was 78 beats per minute (bpm) according to the first main peak in the FFT spectrum, indicating the health state of the volunteer.

In TCM pulse diagnosis, physicians can clearly appreciate the significance of the wrist pulse and correlate it with different diseases by using their fingertips to apply different pressures. ${ }^{43}$ However, this diagnosis relies heavily on an individual's experience and the quality of the sensitive awareness, which affects its reliability and reproducibility. As the value of TCM pulse diagnosis 
become prominent, much effort is required to develop accurate and intelligent pulse diagnosis techniques and instruments. Figure $6 \mathrm{~d}$ shows a schematic of the intelligent pulse diagnosis system, which includes robotic fingers with pressure-sensitive HB electronic skin, artificial intelligence diagnosis, and big data on prior knowledge of pulse diagnosis by TCM physicians; this can be used as an intelligent tool for pulse diagnosis. Given that TCM physicians measure the pulse signal at a certain finger pressure, a pressure sensor with a high sensitivity over a broad range is important for the accurate detection of pulse signals under a high pulse-taking pressure. As the basic function of the intelligent pulse diagnosis system, we simulated the TCM pulse diagnosis process. Dynamic pulse-taking pressure was applied by using fingers on a single region of the radial artery and simultaneously obtaining the pressure signal from the HB sensor. The acquired signal contains the pulse signal and pulse-taking pressure, which are two key factors for evaluating the pulse types. ${ }^{44}$ Figure $6 \mathrm{e}$ shows the obtained signal under a dynamic pulse-taking pressure. Figure $6 \mathrm{f}$ shows the corresponding magnified pulse waves under the four different pulse-taking pressures in Figure 6e. The results showed that all three characteristic peaks could be clearly distinguished, even under heavy pressure (Pressure ${ }_{1}$ ). As shown in Figure 6f, with increasing pulse-taking pressure, the measured pulse amplitudes decreased owing to the reduced amount of blood perfusion. 
(a)

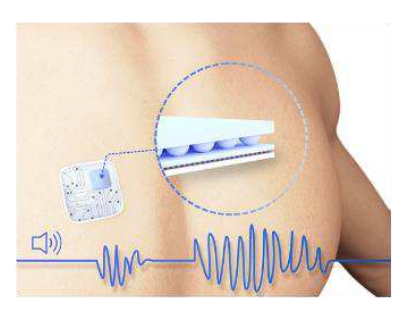

(b)

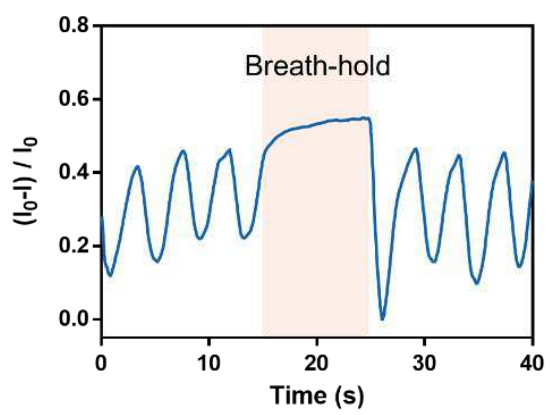

(c)

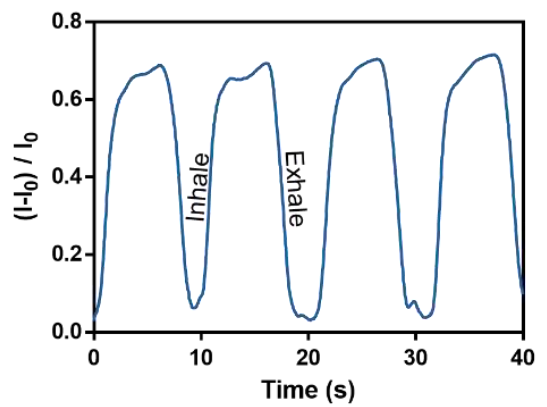

(d)

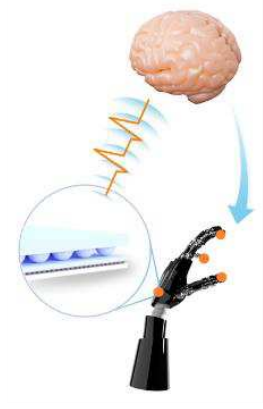

(e)

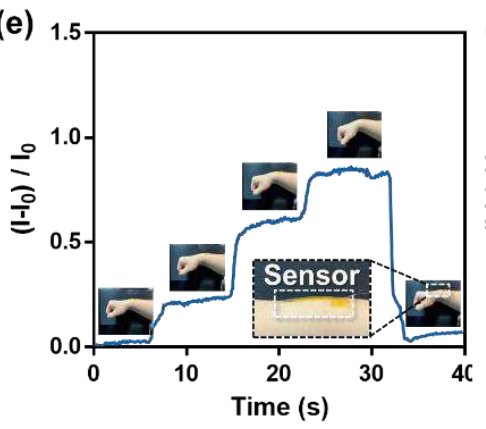

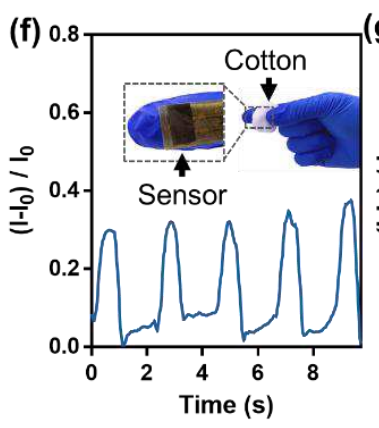

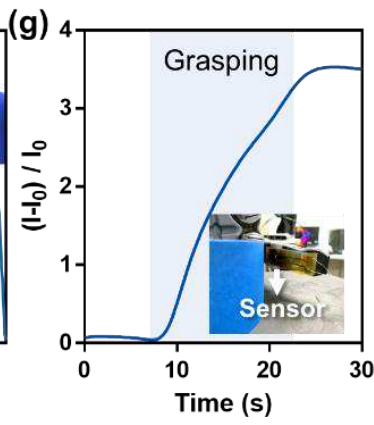

Figure 7. Application of the HB sensor for breath monitoring and electro-tactile feedback. (a)

Breath monitoring schematic showing the HB sensor attached to the chest. (b) Monitored normal breathing and simulated apnea signals. (c) Monitored deep breath signals. (d) Application diagram of intelligent prosthesis. (e) Continuous response of the current of the wrist joint under different bending angles. The inset shows the sensor is attached above the wrist using silica gel. (f) Current variation as a function of time as the volunteer grasps a cotton ball. (g) Measured feedback signal from the HB graphene sensor during the capturing process of the manipulator; inset shows an HB sensor attached to the inside of the manipulator.

Since breathing is a basic physiological activity, its patterns can reflect the physical and mental status of humans, which can also be regulated via deliberate breath control. As a common disease, sleep apnea syndrome may increase the risk of high blood pressure, heart attack, etc. ${ }^{45}$ Thus, continuous monitoring of breath is necessary for the diagnosis and therapy of sleep apnea syndrome. In this study, we attached the HB sensor to the chest of a volunteer for breath monitoring, 
as shown in Figure $7 \mathrm{a}$. The results in Figure $7 \mathrm{~b}$ show that the $\mathrm{HB} \backslash$ sensor can clearly differentiate between normal breaths and simulated apnea, in which a breath hold existed for nearly $10 \mathrm{~s}$ (minimum duration of apnea in apnea syndrome). The monitored normal breathing rate was 18 breaths/min, corresponding to that of healthy adults (12-20 breaths/min) ${ }^{46}$ Moreover, the monitored deep breathing signal in Figure $7 \mathrm{c}$ shows a decrease in breathing rate to 7.5 breaths $/ \mathrm{min}$ and an increase in intensity. The above results indicate the HB sensor's potential in quantifying the subject's respiration status and providing alerts for abnormal breathing conditions.

Our somatosensory system gives us the ability to interact with complex environments through receptors that convert abundant stimuli into action potentials, which are then transmitted by neurons to the brain for decision making. Thus, a pressure-sensitive flexible sensor is necessary for a sensory feedback loop solution in the applications of intelligent prostheses and robotics. ${ }^{17}$ Here we demonstrated the application potential of the HB sensor in intelligent prosthesis, evaluation of joint rehabilitation, joint motion control of manipulators, surgical robots, etc. First, the ability of the HB sensor to detect real-time dynamic joint movements was demonstrated. Figure 7e shows the continuous response curve of the wrist joint under different bending angles. The output current increased as the bending angle increased and remained stable at a certain bending angle. When the wrist joint straightened again, the output response of the sensor returned to its original state. Another common action for a prosthetic limb is object grasping, in which tactile perception is of great importance. To demonstrate the ability of the HB sensor to measure the finger pressure, we measured the tactile force while lifting a soft object (a cotton ball), as shown in Figure 7f. Tactile perception is also required for robots to manipulate objects dexterously and enhance safety in human-robot-environment interactions. Here the potential of the HB sensor to achieve sensing feedback in real time and control a manipulator to grasp an object (a tape) was 
demonstrated (Movie S1, Supporting Information). The HB sensor was attached to the inside of the manipulator allows it to sense external forces and self-monitor its operation stage (Figure $7 \mathrm{~g}$ ).

\section{CONCLUSIONS}

Here, we demonstrated a nature-inspired pressure-sensitive electronic skin devices based on a hierarchical branch microstructure, which is realized using a simple multilayer superposition approach. Such hierarchical branching structure, composed of multi-layer structures with precise dimensions spanning multiple scales is tunable and scalable in structural morphology, size, number of layers and other aspects. FEA results demonstrated that such HB structure can realize the branch transmission of pressure. Given this advantage, the deformation of microstructures is efficiently scheduled, which will effectively optimize the sensitivity and pressure detection range of pressure sensors. Experiments results showed that the pressure sensor with the HB structure exhibits almost 34- and 55-fold improvements in sensitivity over the medium-pressure $(<10 \mathrm{kPa})$ and highpressure $(>10 \mathrm{kPa})$ ranges, respectively. When compared with the previously reported pressure sensors based on multi-layer or hierarchical structure, the proposed HB structure has a more significant effect on optimizing the sensitivity. In addition, we demonstrated its potential in healthcare applications and artificial sensory feedback solutions. The small and thin nature of the sensor enables its application in quantifying medical diagnostic processes, such as intelligent TCM pulse diagnosis, where both high sensitivity and broad dynamic response range are required.

\section{MATERIALS AND METHODS}

Preparation of the PDMS and PDMS/rGO films. The PDMS prepolymer was prepared by mixing the base silicone gel well with a crosslinker (Dow Corning Sylgard 184) in a weight ratio of 10:1, followed by degassing in vacuum to remove bubbles. Subsequently, the prepared precursor 
was spin-coated onto the micropatterned mold. Then, a microdome-structured film was obtained after curing the above precursor on a hot plate at a temperature of $80{ }^{\circ} \mathrm{C}$ for $1 \mathrm{~h}$ and carefully peeling it off from the mold. The GO solution (Nanjing XFNANO, Materials Tech Co., Ltd.) was fully mixed with tetrahydrofuran (Macklin Inc., China) at a volume ratio of 1:5 to enhance the dispersion of GO. Then, the PDMS film was treated with oxygen plasma to improve its surface hydrophilicity. Subsequently, the prepared GO solution was spray-coated on the PDMS surface and dried at $80^{\circ} \mathrm{C}$ at least three times to obtain a uniform GO layer. Finally, the PDMS/GO samples were heated at $200^{\circ} \mathrm{C}$ for $2 \mathrm{~h}$ to reduce GO.

Preparation of the electrode layer. A $50-\mu \mathrm{m}$ thick polyethylene (PEN) film (Teijing-Dupont) was used as the electrode substrate. Using an electronic circuit printer (BroadJET L3000table, Beijing BroadTeko Technology Co., Ltd.) with a 100- $\mu \mathrm{m}$ line width, a nano silver conductive ink (CON-INK550, Beijing BroadTeko Technology Co., Ltd.) was printed on the PEN substrates. The printed electrodes were then treated at $150{ }^{\circ} \mathrm{C}$ for $30 \mathrm{~min}$ to enhance the conductivity.

Assembly of the HB graphene sensor. The HB graphene sensor consists of three parts, including one PDMS layer with larger microdomes serving as the primary layer, one PDMS/rGO layer with smaller microdomes serving as the secondary and piezoresistive layer, and one printed side-by-side electrode layer. First, the as-prepared PDMS film and the PDMS/rGO conductive film were cut to the desired size. Then, the two micro-dome-patterned substrates with different microdome sizes were treated with oxygen plasma and bonded together. Finally, the hierarchical layer was fixed on the electrode layer using Kapton tape. For the monolayer structure pressure sensor, only one microdome-patterned PDMS/rGO layer was stacked on the electrodes and encapsulated in the same way.

Characterization and measurement of the HB graphene sensor. The microstructures and 
cross-sections of the hierarchical branching structure were characterized via field-emission SEM (Gemini, Zeiss). The images of the structured PDMS/rGO (secondary layer) were obtained clearly without Au sputtering, while the images of the structured PDMS (primary layer) and the crosssectional images were obtained with Au sputtering. Raman spectra were obtained using a laser with a wavelength of $514 \mathrm{~nm}$ (LabRam-HR, HORIBA Jobin Yvon). Optical micrographs were

obtained using an optical microscope (IX73, Olympus). To measure the pressure-sensing performance of the pressure sensor, a force was applied using a universal testing machine (ZQ990B, ZhiQu Precision Instrument), while the electrical signals of the pressure sensors were collected using a sampling multimeter (DMM7510, Keithley). The pressure sensor was attached to the inside of a robotic gripper's fingers (JACO2-KG3, Kinova). FEA was performed using COMSOL Multiphysics. Participants took part in experiments described herein with informed consent and no formal approval from these experiments was required.

\section{ASSOCIATED CONTENT}

\section{Supporting Information}

The following files are available free of charge.

Additional information and figures (PDF)

Demonstration of the HB sensor for force feedback control of a manipulator (MP4)

\section{NOTES}

The authors declare no competing financial interests.

\section{REFERENCES}

1. Wegst, U. G. K. et al. Bioinspired structural materials. Nature Materials. 14, 23-36 (2015).

2. Liu, Z. et al. Functional gradients and heterogeneities in biological materials: Design principles, 
functions, and bioinspired applications. Progress in Materials Science. 88, 467-498 (2017).

3. Liu, K. \& Jiang, L. Bio-inspired design of multiscale structures for function integration. Nano Today. 6, 155-175 (2011).

4. West, G. B. et al. A General Model for the Origin of Allometric Scaling Laws in Biology. Science. 276, 122-126 (1997).

5. Shan, X. D. et al. Geometry Optimization of Self-Similar Transport Network. Mathematical Problems in Engineering. 2011, 421526 (2011).

6. McCulloh, K. A. et al. Water transport in plants obeys Murray's law. Nature. 421, 939-942 (2003).

7. Murray, C. D. The Physiological Principle of Minimum Work. I. The Vascular System and the Cost of Blood Volume. 12, 207-214 (1926).

8. Sherman, T. F. On connecting large vessels to small. The meaning of Murray's law. Journal of General Physiology. 78, 431-453 (1981).

9. Delmas, P. et al. Molecular mechanisms of mechanotransduction in mammalian sensory neurons. Nature reviews. Neuroscience. 12, 139-153 (2011).

10. Ruth, S. R. A. et al. Microengineering Pressure Sensor Active Layers for Improved Performance. Advanced Functional Materials. 30, 2003491 (2020).

11. Chu, Y. et al. Human Pulse Diagnosis for Medical Assessments Using a Wearable Piezoelectret Sensing System. Advanced Functional Materials. 28, 1803413 (2018).

12. Chen, Y. et al. Skin-like biosensor system via electrochemical channels for noninvasive blood glucose monitoring. Science Advances. 3, e1701629 (2017).

13. Wang, C. et al. Monitoring of the central blood pressure waveform via a conformal ultrasonic device. Nature Biomedical Engineering. 2, 687-695 (2018).

14. Beker, L. et al. A bioinspired stretchable membrane-based compliance sensor. Proceedings of the National Academy of Sciences. 117, 11314-11320 (2020).

15. Dagdeviren, C. et al. Conformable amplified lead zirconate titanate sensors with enhanced piezoelectric response for cutaneous pressure monitoring. Nature Communications. 5, 4496 (2014).

16. Boutry, C. M. et al. A Sensitive and Biodegradable Pressure Sensor Array for Cardiovascular Monitoring. Advanced Materials. 27, 6954-6961 (2015).

17. Xu, K. et al. Flexible Hybrid Sensor Systems with Feedback Functions. Advanced Functional 
Materials. n/a, 2007436.

18. Rus, D. \& Tolley, M. T. Design, fabrication and control of soft robots. Nature. 521, 467-475 (2015).

19. Wang, X. et al. Flexible Sensing Electronics for Wearable/Attachable Health Monitoring. Small. 13, 1602790 (2017).

20. Jayathilaka, W. A. D. M. et al. Significance of Nanomaterials in Wearables: A Review on Wearable Actuators and Sensors. Advanced Materials. 31, 1805921 (2019).

21. Park, J. et al. Giant Tunneling Piezoresistance of Composite Elastomers with Interlocked Microdome Arrays for Ultrasensitive and Multimodal Electronic Skins. ACS Nano. 8, 46894697 (2014).

22. Park, J. et al. Fingertip skin-inspired microstructured ferroelectric skins discriminate static/dynamic pressure and temperature stimuli. Science Advances. 1, e1500661 (2015).

23. Wang, Z. et al. The Semiconductor/Conductor Interface Piezoresistive Effect in an Organic Transistor for Highly Sensitive Pressure Sensors. Advanced Materials. 31, 1805630 (2019).

24. Zhu, B. et al. Microstructured Graphene Arrays for Highly Sensitive Flexible Tactile Sensors. Small. 10, 3625-3631 (2014).

25. Park, H. et al. Stretchable Array of Highly Sensitive Pressure Sensors Consisting of Polyaniline Nanofibers and Au-Coated Polydimethylsiloxane Micropillars. ACS Nano. 9, 9974-9985 (2015).

26. Pyo, S. et al. Multi-Layered, Hierarchical Fabric-Based Tactile Sensors with High Sensitivity and Linearity in Ultrawide Pressure Range. Advanced Functional Materials. 29, 1902484 (2019).

27. Kang, S. et al. Highly Sensitive Pressure Sensor Based on Bioinspired Porous Structure for Real-Time Tactile Sensing. Advanced Electronic Materials. 2, 1600356 (2016).

28. Ge, G. et al. A flexible pressure sensor based on $\mathrm{rGO} /$ polyaniline wrapped sponge with tunable sensitivity for human motion detection. Nanoscale. 10, 10033-10040 (2018).

29. Pang, Y. et al. Epidermis Microstructure Inspired Graphene Pressure Sensor with Random Distributed Spinosum for High Sensitivity and Large Linearity. ACS Nano. 12, 2346-2354 (2018).

30. Wang, Z. et al. High Sensitivity, Wearable, Piezoresistive Pressure Sensors Based on Irregular Microhump Structures and Its Applications in Body Motion Sensing. Small. 12, 3827-3836 
(2016).

31. Wan, Y. et al. A Highly Sensitive Flexible Capacitive Tactile Sensor with Sparse and HighAspect-Ratio Microstructures. Advanced Electronic Materials. 4, 1700586 (2018).

32. Lee, Y. et al. Bioinspired Gradient Conductivity and Stiffness for Ultrasensitive Electronic Skins. ACS Nano. 15, 1795-1804 (2021).

33. Mannsfeld, S. C. B. et al. Highly sensitive flexible pressure sensors with microstructured rubber dielectric layers. Nature Materials. 9 , 859 (2010).

34. Schniepp, H. C. et al. Functionalized Single Graphene Sheets Derived from Splitting Graphite Oxide. The Journal of Physical Chemistry B. 110, 8535-8539 (2006).

35. Cai, D. et al. Raman, mid-infrared, near-infrared and ultraviolet-visible spectroscopy of PDMS silicone rubber for characterization of polymer optical waveguide materials. Journal of Molecular Structure. 976, 274-281 (2010).

36. Chen, W. \& Yan, L. Preparation of graphene by a low-temperature thermal reduction at atmosphere pressure. Nanoscale. 2, 559-563 (2010).

37. Tian, H. et al. Wafer-Scale Integration of Graphene-based Electronic, Optoelectronic and Electroacoustic Devices. Scientific Reports. 4, 3598 (2014).

38. Lee, Y. et al. Flexible Ferroelectric Sensors with Ultrahigh Pressure Sensitivity and Linear Response over Exceptionally Broad Pressure Range. ACS Nano. 12, 4045-4054 (2018).

39. Wu, W. et al. Taxel-Addressable Matrix of Vertical-Nanowire Piezotronic Transistors for Active and Adaptive Tactile Imaging. Science. 340, 952-957 (2013).

40. Nichols, W. W. Clinical measurement of arterial stiffness obtained from noninvasive pressure waveforms. American Journal of Hypertension. 18, 3S-10S (2005).

41. Tomiyama, H. et al. Arterial stiffness and pressure wave reflection in the development of isolated diastolic hypertension. Journal of hypertension (2020).

42. Yang, T. et al. A Wearable and Highly Sensitive Graphene Strain Sensor for Precise HomeBased Pulse Wave Monitoring. ACS Sensors. 2, 967-974 (2017).

43. Shu, J.-J. \& Sun, Y. Developing classification indices for Chinese pulse diagnosis. Complementary Therapies in Medicine. 15, 190-198 (2007).

44. Jin, C. et al. A Wearable Combined Wrist Pulse Measurement System Using Airbags for Pressurization. Sensors. 19 (2019).

45. Chen, Y. et al. Skin-Like Hybrid Integrated Circuits Conformal to Face for Continuous 
Respiratory Monitoring. Advanced Electronic Materials. 6, 2000145 (2020).

46. Lovett, P. B. et al. The vexatious vital: Neither clinical measurements by nurses nor an electronic monitor provides accurate measurements of respiratory rate in triage. Annals of Emergency Medicine. 45, 68-76 (2005). 


\section{Supporting Information}

\section{A Nature-inspired Hierarchical Branching Structure \\ Pressure Sensor for Medical Wearables}


Section S1. Analysis of the relation between the microdome sizes of primary and secondary layer for maximum performance (sensitivity and linearity).

In order to further investigate the relation between the microdome sizes of primary and secondary layer on sensors performance, the performance of sensors with different size parameters was compared and analyzed through FEA simulation. The diameter of the microdomes in the primary layer was kept at $50 \mu \mathrm{m}$, and the diameter of the microdomes in the secondary layer was adjusted from $3 \mu \mathrm{m}$ to $51 \mu \mathrm{m}$ (the increment is $2 \mu \mathrm{m}$ ). The edge distance of adjacent microdomes in the secondary layer was kept at $2 \mu \mathrm{m}$. Figure S1a shows the relative current variation of the sensors with different microdome radius in the secondary layer in response to the stress. To further analyze the relation between the microdome radius of primary and secondary layer, we made a linear fitting of each curve in Figure S1a and extracted the slope $(k)$ and the $R$ square $\left(R^{2}\right)$ of the fitting line to represent sensitivity and linearity of the sensor, respectively. As shown in Figure $\mathrm{S} 1 \mathrm{c}$, with the increase of the microdome radius in the secondary layer, $\mathrm{k}$ increased at first and then rapidly decreased to a relatively stable value, and $\mathrm{R}^{2}$ decreased monotonically.

In order to make the conclusion more general, the radius of the microdomes in two layers is normalized. According to the HB structure proposed in this work, the number $(\mathrm{N})$ of secondary microdomes that can be covered by a primary structure can be expressed as:

$$
\mathrm{N}=\frac{\pi r_{1}^{2}}{\left(2 r_{2}+d\right)^{2}}
$$

where $r_{1}, r_{2}$ represented the radius of the microdomes in the primary and secondary layer, and $d$ represents the edge distance of adjacent microdomes in the secondary layer. The total area of secondary structures that can be covered by a single primary structure is $S_{2}=\mathrm{N} \cdot \pi r_{2}^{2}$. The ratio of $\mathrm{S}_{2}$ to the area of a single primary structure can be expressed as: 


$$
\text { Ratio }=\frac{N \cdot \pi r_{2}^{2}}{\pi r_{1}^{2}}=N \frac{r_{2}^{2}}{r_{1}^{2}}=\pi \frac{1}{\left(2+\frac{d}{r_{2}}\right)^{2}}
$$

As shown in Figure S1d, when the ratio was greater than 0.7 , both sensitivity and linearity attenuated rapidly, which revealed 0.7 might be optimal solution for the maximum performance under the conditions discussed in this section. As can be seen from Figure S1a and e, smaller or more secondary structures improve the linearity of the sensor due to the faster compensating for deformation saturation. However, due to the elastic deformation range of small structure is relatively narrow, its advantage in improving the sensitivity is small. Based on this result, we concluded that there exists an optimal relationship between the microdome sizes of primary and secondary layer for maximum performance. The discussion in this section is one feature case, which can be used as a guide for the design of HB sensors. 

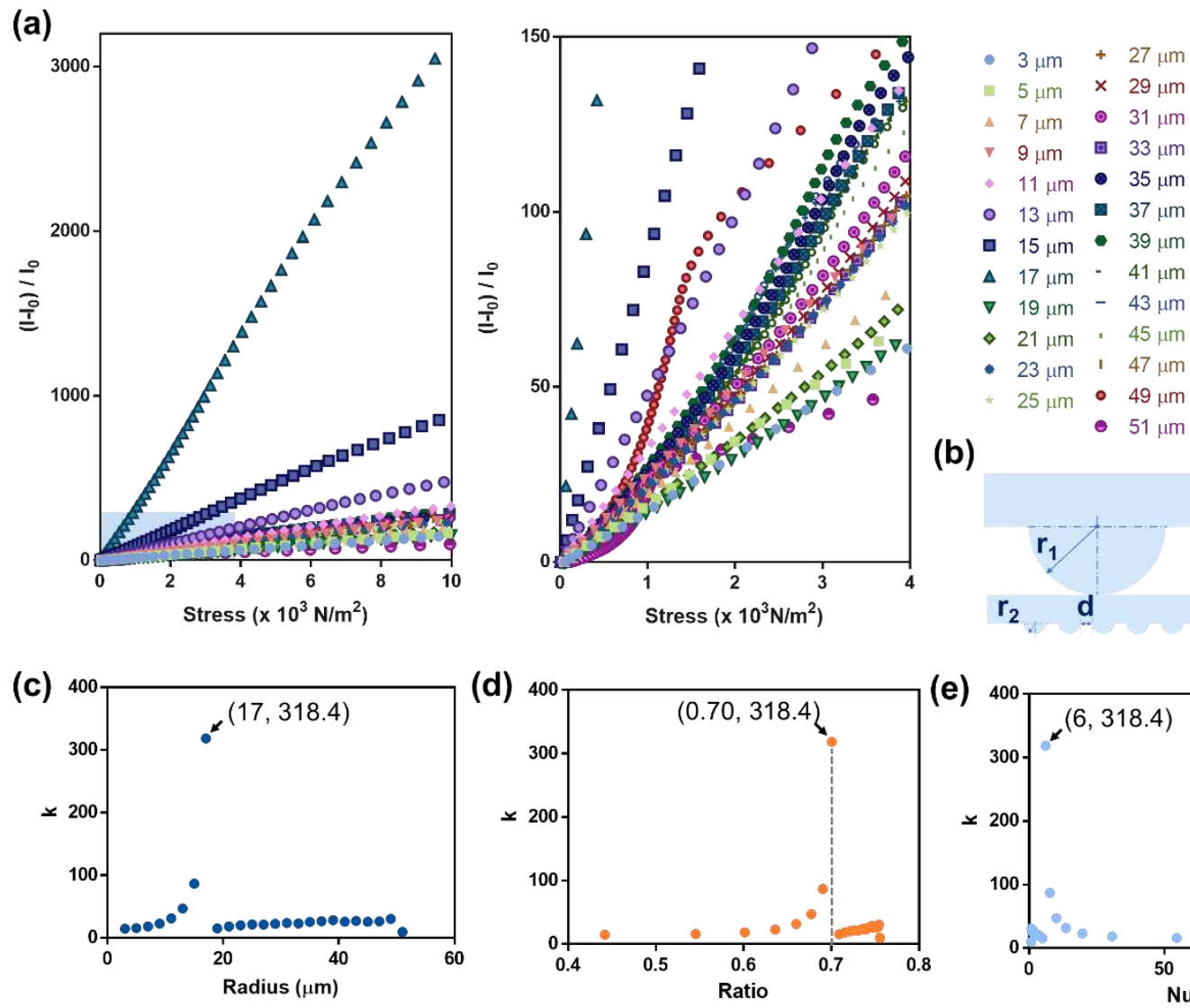

(e)
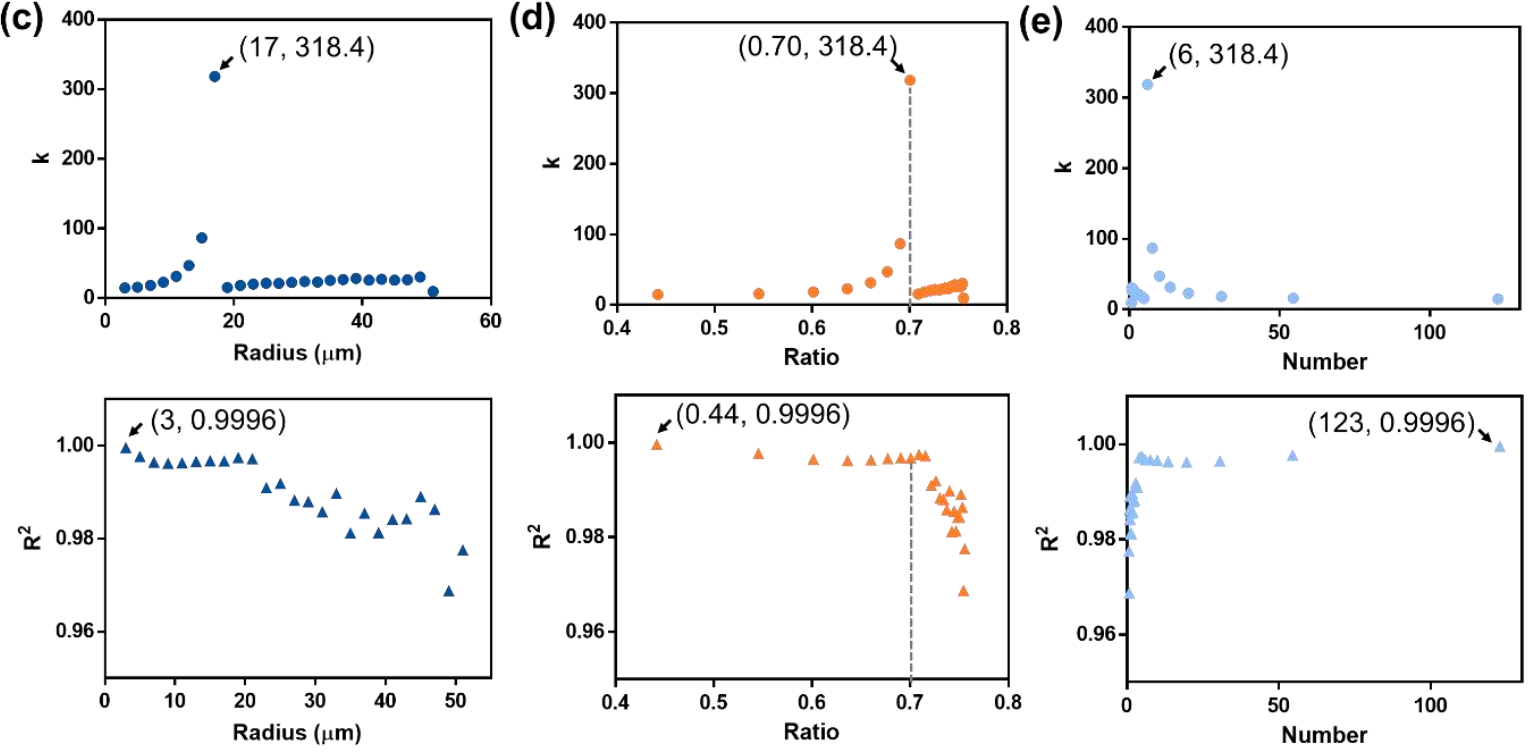

Figure S1. (a) Relative current variation under various stress and the corresponding magnified graph for the sensors with different microdome radius in the secondary layer. (b) Diagram of Dimension Labeling. (c) The slope $\mathrm{k}$ and the $\mathrm{R}$ square of linear fitting lines of each curve in Figure S2a versus the microdome radius, (d) the area ratio and (e) the microdome number. 
Section S2. Analysis of the circuit model.

To systematically understand the resistance variation during the loading process, the circuit model of a pressure sensor with side-by-side electrode configuration and monolayer periodic microarray structure (Figure S7) has been described as:

$$
\mathrm{R}_{\text {device }}=2 \mathrm{R}_{\text {single }}+\mathrm{R}_{\text {gap }}=\frac{2}{\mathrm{n} \cdot \frac{1}{\mathrm{R}_{\text {contact }}}}+\mathrm{R}_{\text {gap }}
$$

where $\mathrm{R}_{\text {device, }} \mathrm{R}_{\text {single, }}$ and $\mathrm{R}_{\text {gap }}$ are the total resistance of the device, the complex resistances between the conductive microstructure and the electrode and a gap resistance between the two side-by-side electrodes, respectively. Furthermore, $\mathrm{R}_{\text {single }}$ can be expressed as the sum of all the microstructure contact resistances $\left(\mathrm{R}_{\text {contact }}\right)$ connected in parallel, and $\mathrm{n}$ is the number of microstructures in contact with the electrode. It's worth noting that because of the existence of cracks between the rGO, $\mathrm{R}_{\text {contact }}$ should be further calculated as follows:

$$
\mathrm{R}_{\text {contact }}=\frac{\mathrm{R}_{\mathrm{c} 1} \mathrm{R}_{\mathrm{c} 2}}{\mathrm{R}_{\mathrm{c} 1}+\mathrm{R}_{\mathrm{c} 2}}
$$

where $R_{c 1}$ and $R_{c 2}$ are the resistance of contact interfaces and the resistance in the rGO cracks, respectively. At lower pressure, $\mathrm{R}_{\mathrm{c} 1}$ dominates the total resistance due to the sharp increase of the contact interface, although $\mathrm{R}_{\mathrm{c} 2}$ also contributes to the resistance variation. With increasing pressure, $\mathrm{R}_{\mathrm{c} 2}$ will be saturated. Thus, the equation can be simplified as follows:

$$
\mathrm{R}_{\text {device }}=2 \mathrm{R}_{\text {single }}+\mathrm{R}_{\text {gap }}=\frac{2 \mathrm{R}_{\mathrm{c} 1}}{\mathrm{n}}+\mathrm{R}_{\text {gap }}
$$

Overall, $\mathrm{R}_{\mathrm{gap}}$ is related to the gap distance between the two electrodes. When the graphene coated microstructures are in contact with electrodes under external pressure, it can be considered as a constant for a given configuration. Thus, the total resistance of the $\mathrm{R}_{\text {device }}$ is 
dominated by $\mathrm{R}_{\mathrm{cl}}$, which is inversely proportional to the contact area (A) between the conductive microstructure and the bottom flat electrode.
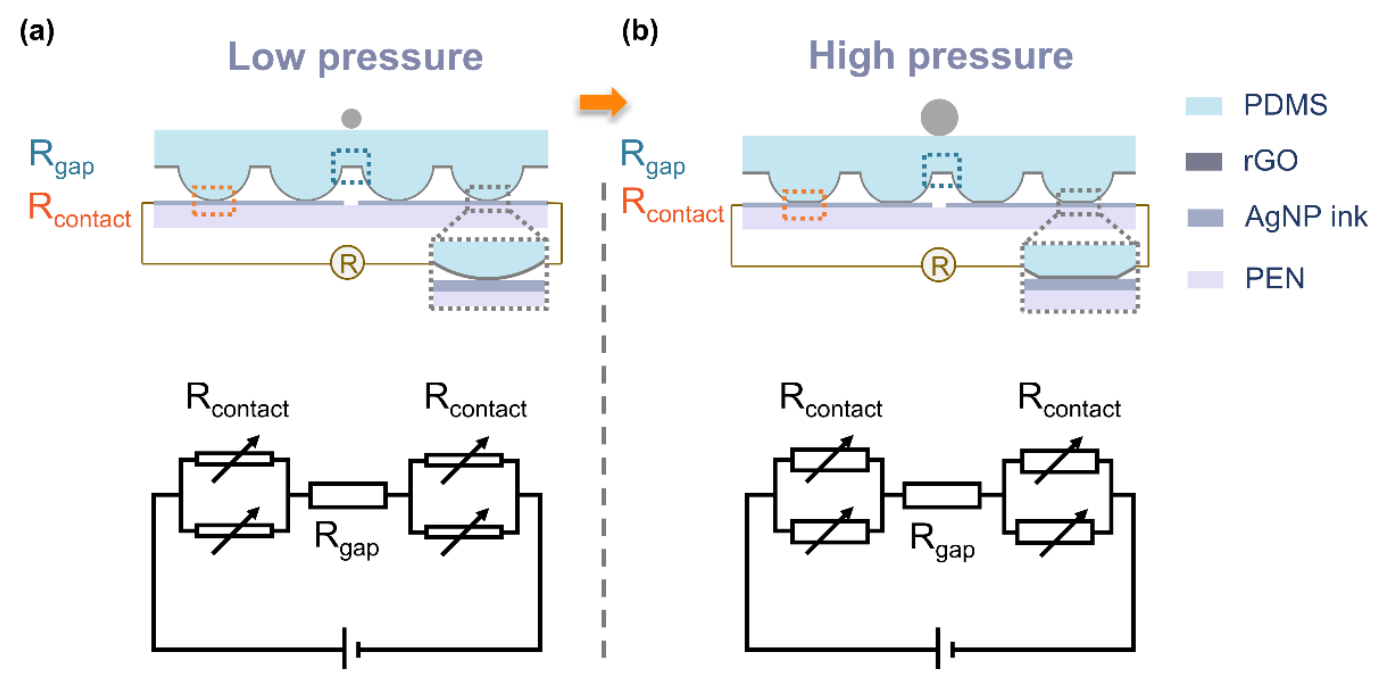

Figure S2. Schematic illustrations showing resistance models of pressure sensors with side-byside electrode configuration and monolayer periodic microarray structure under (a) a low pressure and (b) high pressure. 


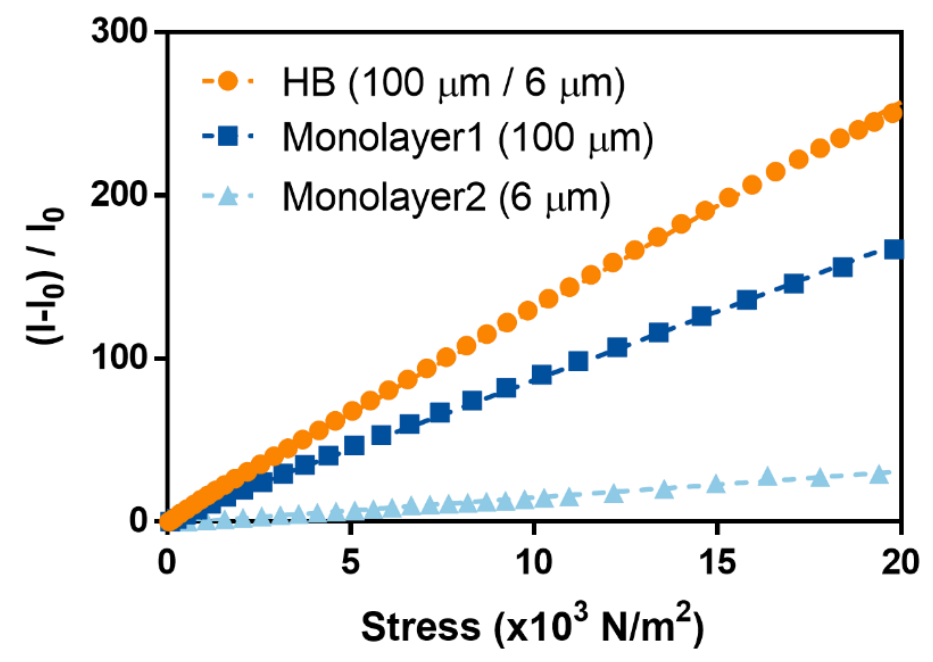

Figure S3. Simulation results of the current variation versus stress for the sensors with different structure. The current variation of the pressure sensor with the HB structure is more rapid, which indicates the enhanced sensitivity by HB structure. 
(a)

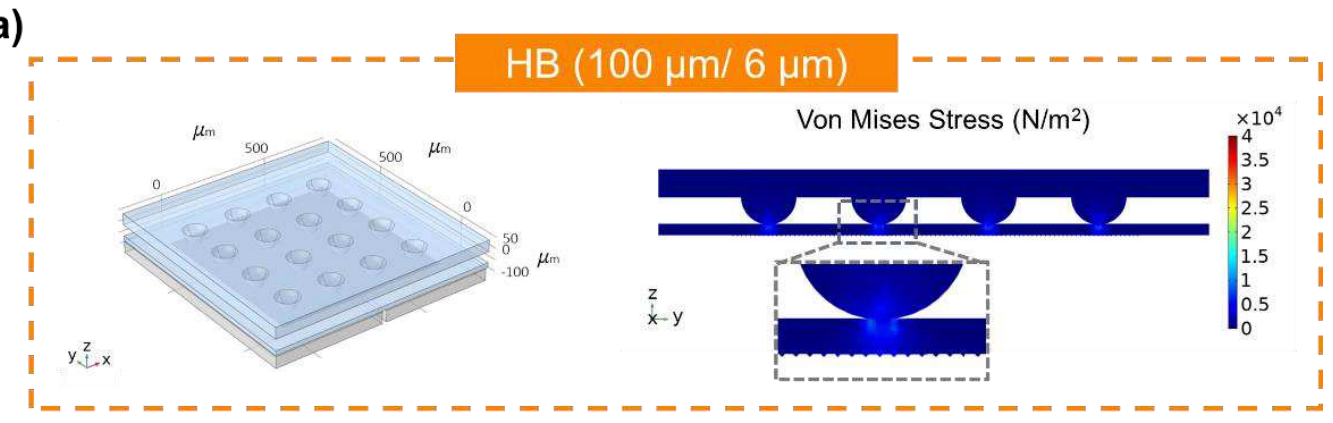

(b)

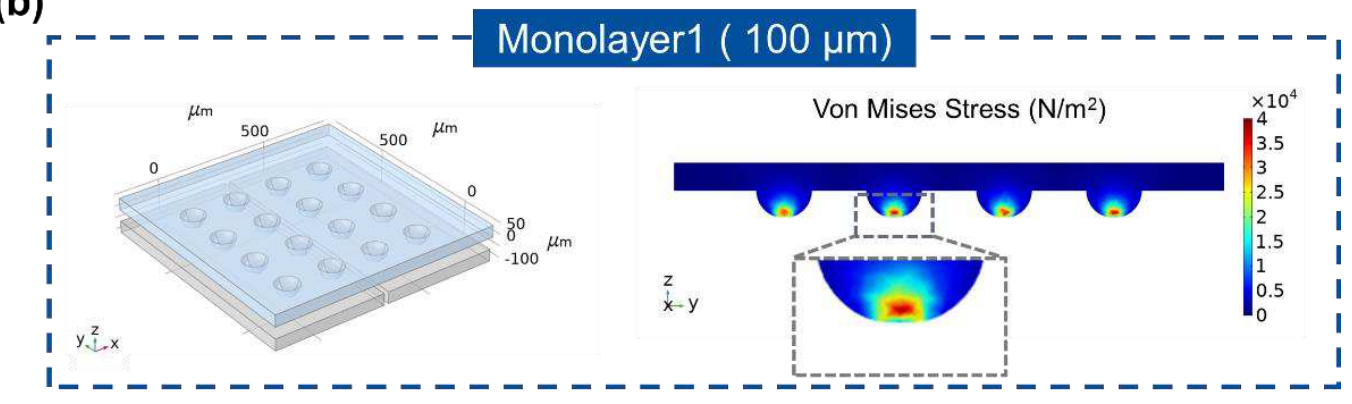

(c)

\section{Monolayer2 ( 6 um)}
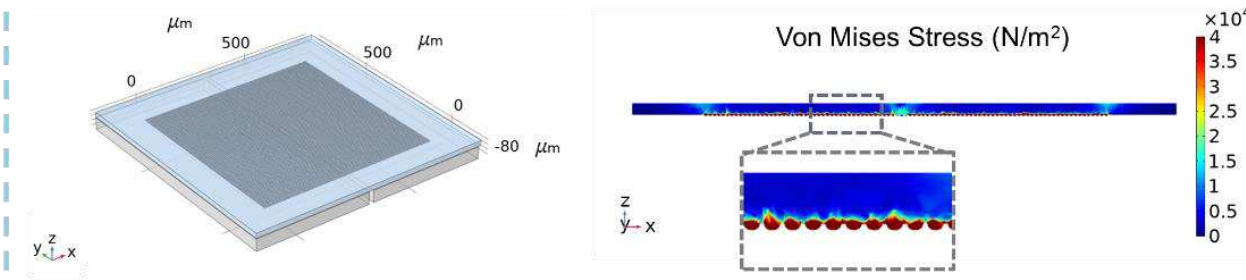

Figure S4. 3D models in the FEA software and the von Mises equivalent stress distribution at a prescribed displacement of $3 \mu \mathrm{m}$ for the three microstructures: (a) HB structure; (b) and (c) monolayer periodic microarray structures. The feature sizes of the above three structures are presented in Table S2. 

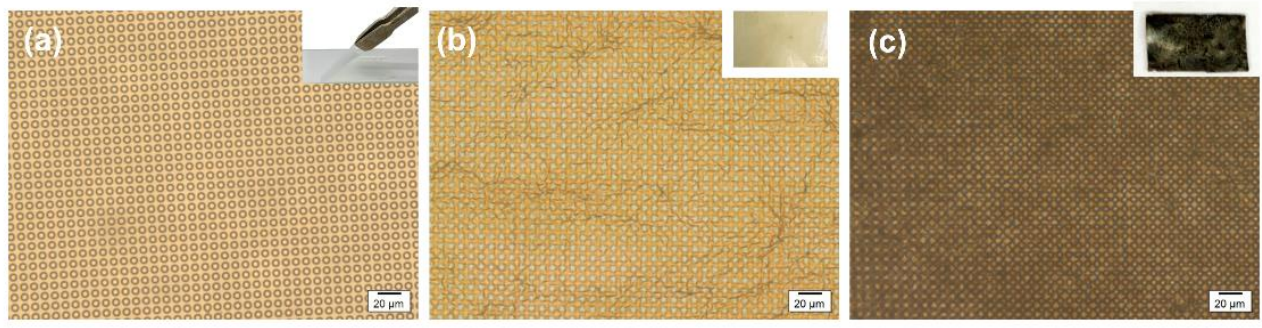

Figure S5. Optical micrographs and photographs (insets) of (a) micro-structured PDMS film, (b) GO-coated PDMS film, and (c) high-temperature-reduced PDMS/rGO film, which prove that the structure of the PDMS film is homogenous and successfully covered by monolayer rGO. 

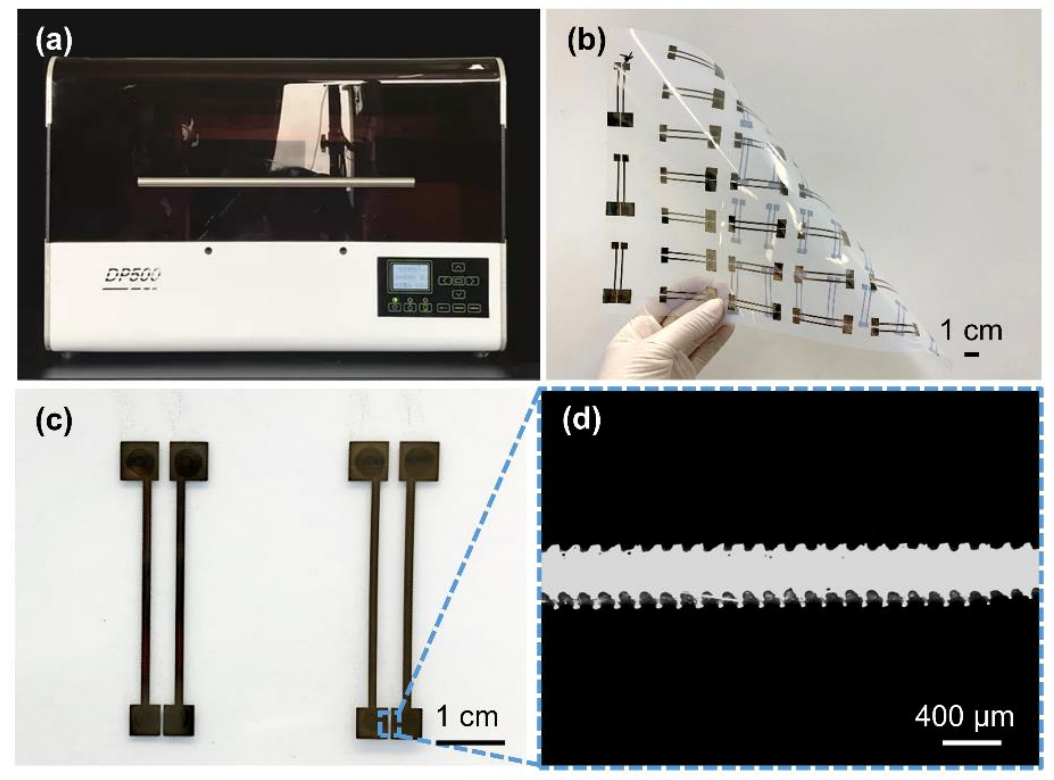

Figure S6. (a) Photograph of the electronic circuit printer. (b) Photograph of the flexible side-byside electrodes. (c) Optical image and (d) corresponding magnified optical image of the side-byside electrodes, which are $6 \mathrm{~mm} \times 6 \mathrm{~mm}$ in size, with a $500 \mu \mathrm{m}$ gap. 

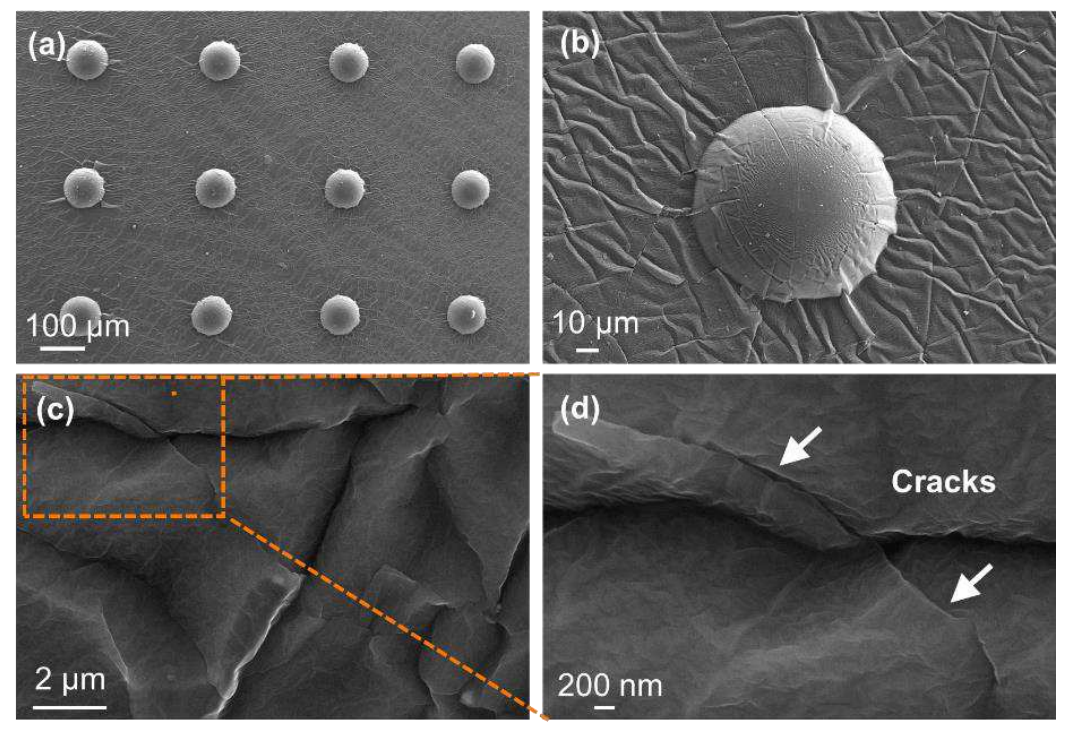

Figure S7. (a) Plane magnified SEM image and (b) corresponding magnified SEM image of the PDMS/rGO film with structural parameters same as those of the primary layer. (c) Highly magnified SEM image and (d) corresponding magnified SEM image of the PDMS/rGO film. 
(a)

(b)

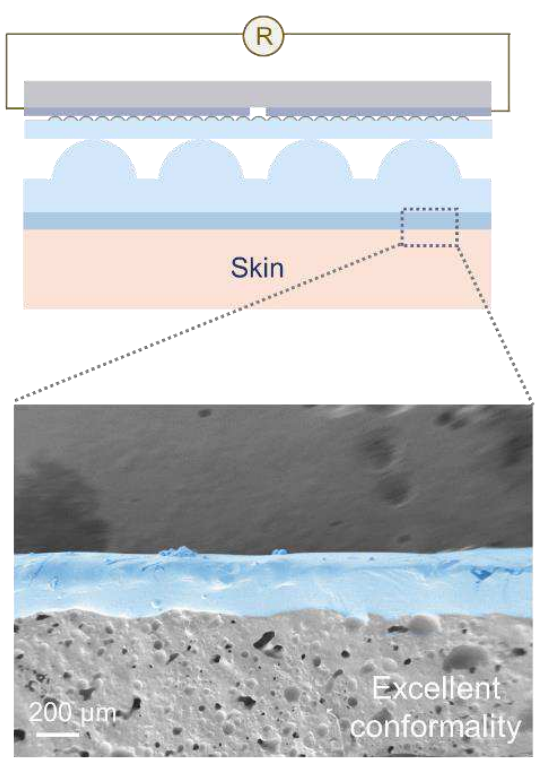

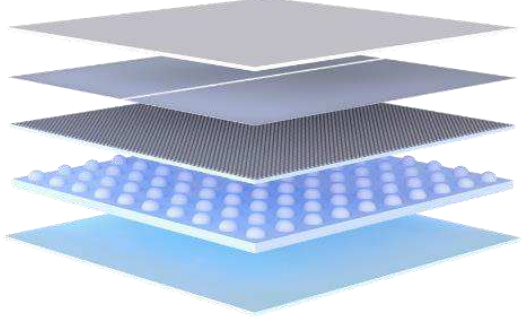

(c)

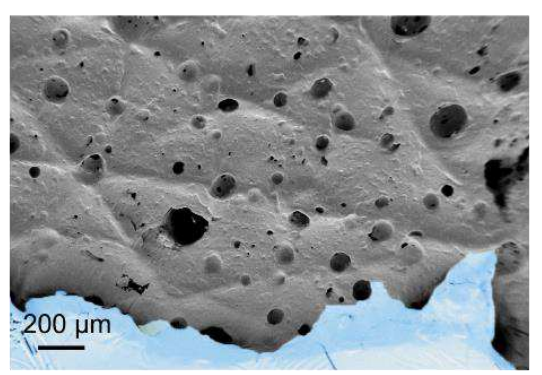

Figure S8. (a) Schematic diagram of the HB graphene sensor combined with silica gel for selfadhering to skin. (b) Cross-sectional and (c) vertical view SEM images showing the degree of conformal contact between a silicone replica (Body Double Silk) of the surface of the skin (grey) and a silica gel (blue) membrane. The thickness of the silica gel layer is $\sim 200 \mu \mathrm{m}$. Bubbles are introduced when stirring the silicone prepolymer. 
Table S1. Structure information of the sensors.

\begin{tabular}{ccccc}
\hline Sensor & Feature & $\begin{array}{c}\text { Diameter } \\
(\boldsymbol{\mu m})\end{array}$ & $\begin{array}{c}\text { Separation } \\
\text { distance }(\boldsymbol{\mu m})\end{array}$ \\
\hline HB & Primary structural layer & Microdomes & 100 & 300 \\
\cline { 2 - 5 } & Secondary structural layer & Microdomes & 6 & 8 \\
\hline Monolayer 1 & Microdomes & 100 & 300 \\
\hline Monolayer 2 & Microdomes & 6 & 8 \\
\hline
\end{tabular}




\section{Figures}

(a)

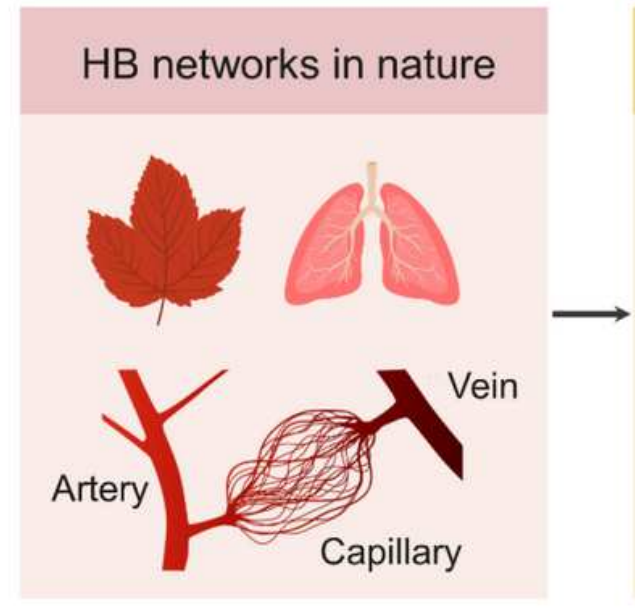

(b)

Structural features of
HB networks in nature

(c)

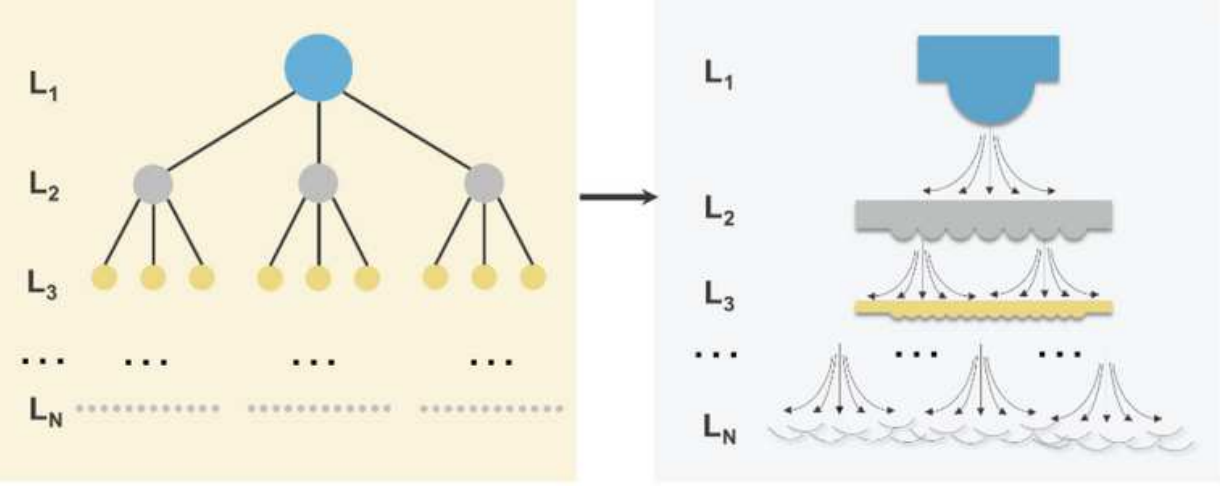

\section{Figure 1}

Hierarchical branching networks. (a) Material transport HB networks in nature. (b) Structural features of HB networks in nature. (c) Concept of the nature-inspired HB structure pressure sensor. 
(a)

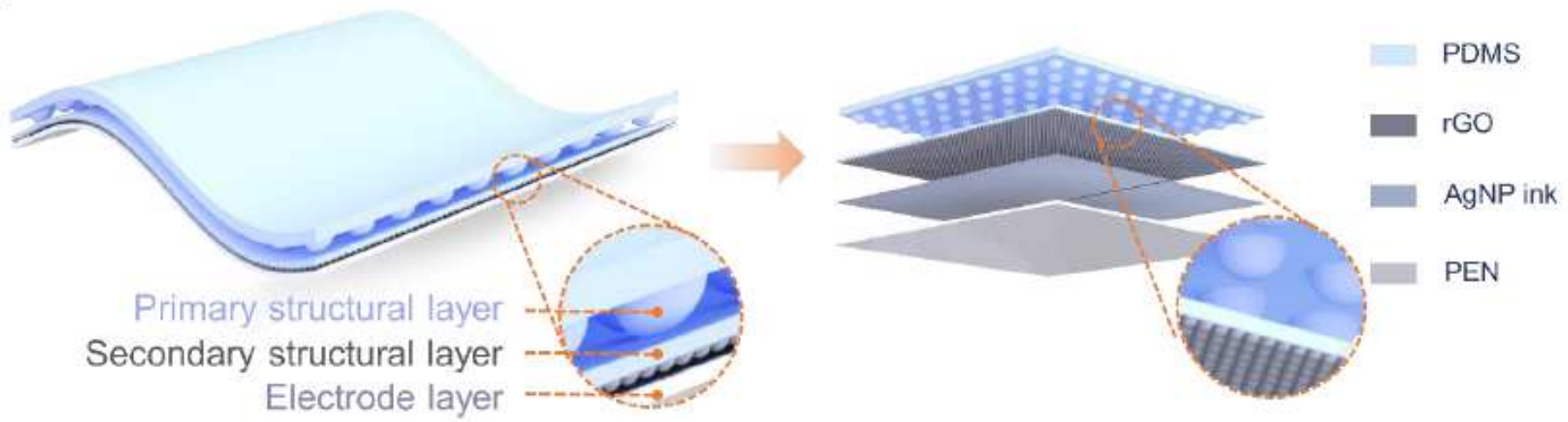

(b)
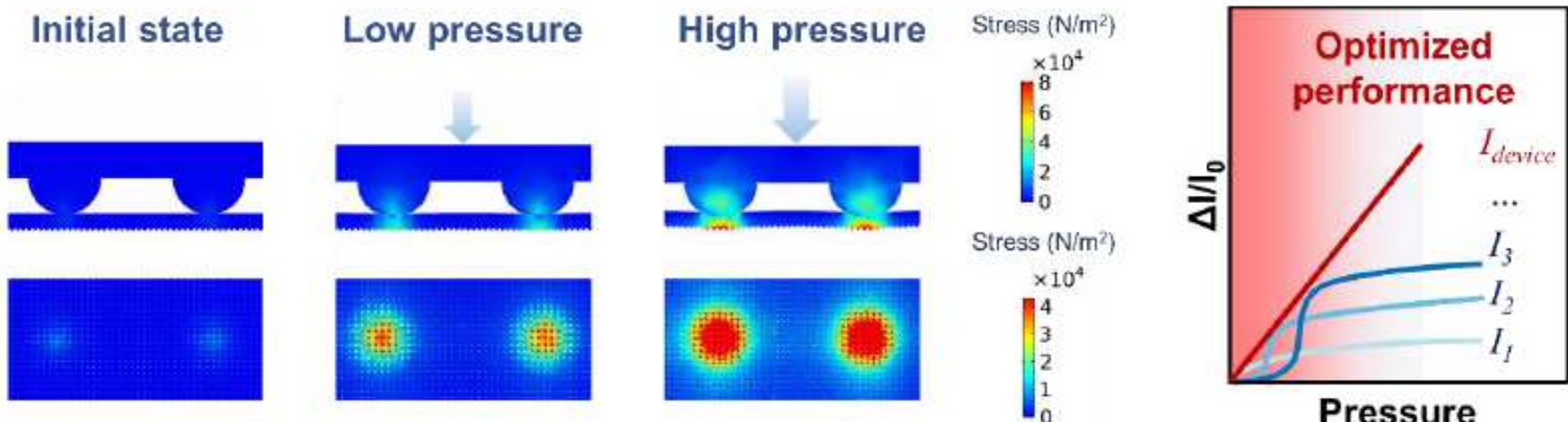

Pressure

(c)
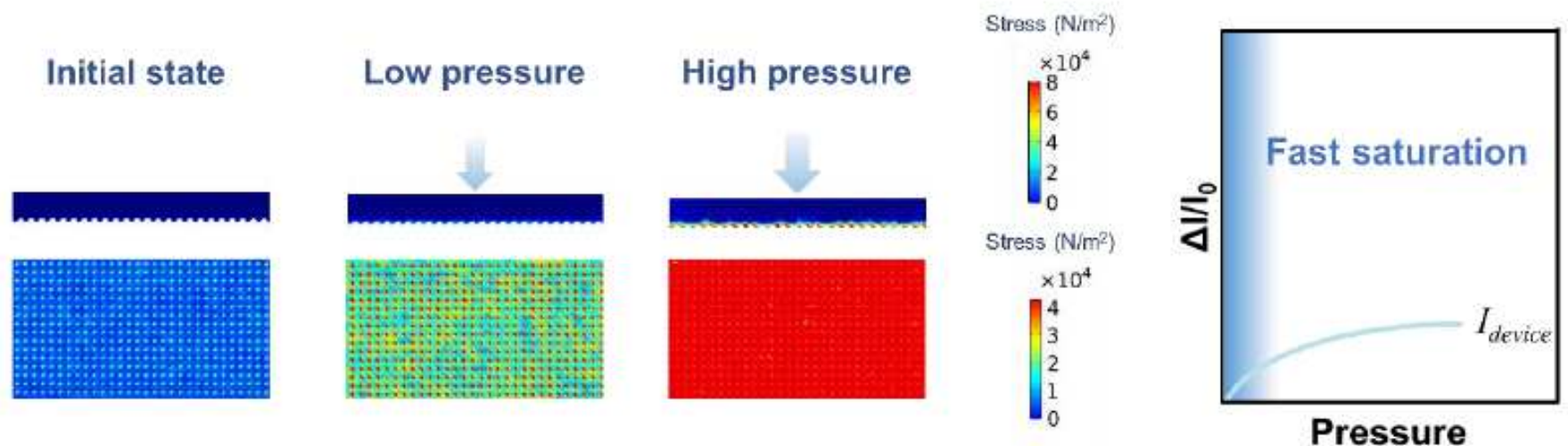

Figure 2

Schematics of the HB pressure sensor. (a) Schematic diagram of the pressure sensor which contains two pressure-sensitive microdome-structured layers and a side-by-side electrodes layer. (b) Deformation and surface von Mises stress of pressure sensors with HB structure. This illustration shows the operating principle of the HB structure. Under applied pressure, the number of contacting microstructures increases, which contributes to improving the sensitivity and linearity range. (c) Deformation and surface von Mises stress of pressure sensors with monolayer regular periodic microstructures. 
(a)
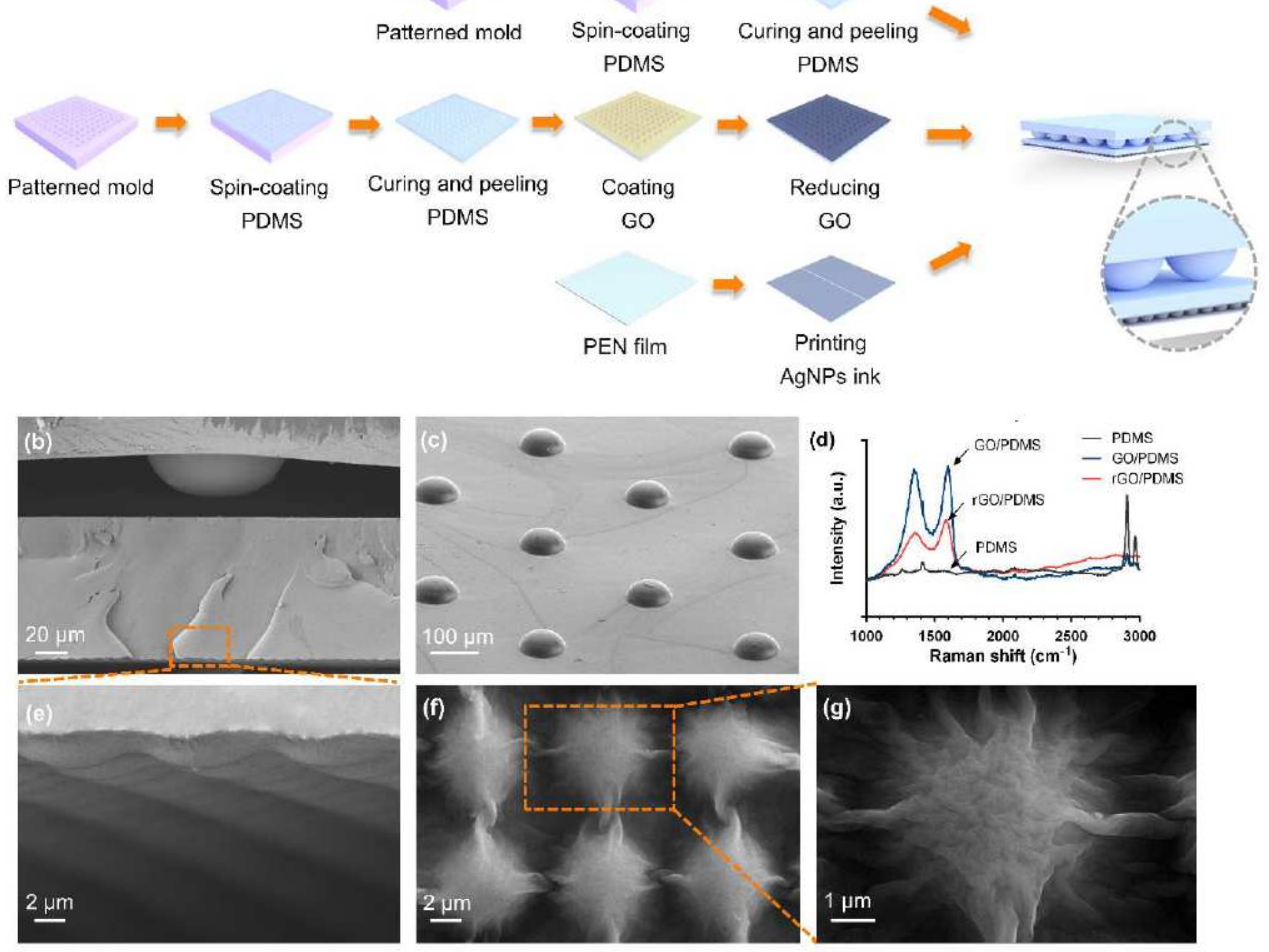

\section{Figure 3}

Fabrication and characterization of the prepared HB graphene sensors. (a) Schematic illustration of the overall fabrication process. (b) Cross-sectional SEM image of the primary layer and secondary layer. (c) Tilted SEM image of the micro-dome array of the PDMS primary layer. (d) Raman spectra of the PDMS, PDMS/GO, and PDMS/rGO. (e) Corresponding magnified SEM image of the secondary layer in (b). (f) Plane magnified SEM image and (g) corresponding magnified SEM image of the PDMS/rGO secondary structural layer. 
(a)

\section{Prescribed displacement}

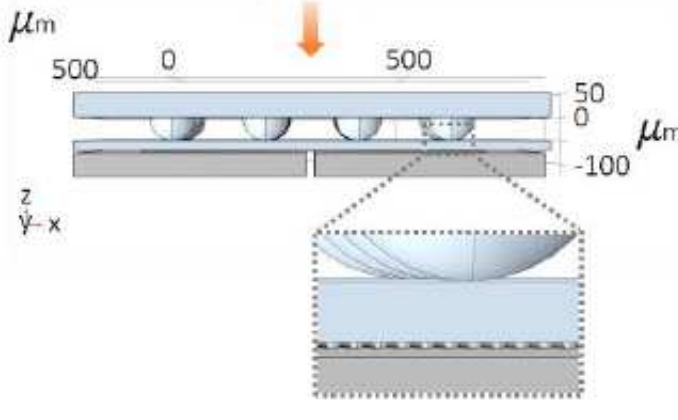

(b)

\section{Prescribed displacement}

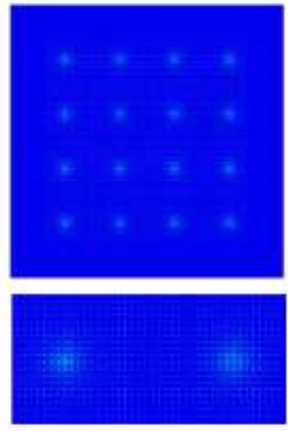

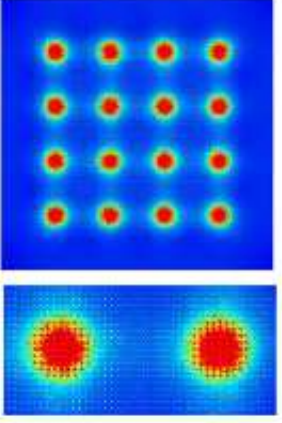

Von Mises Stress $\left(\mathrm{N} / \mathrm{m}^{2}\right)$

$\times 10^{4}$
-4
3
2
1
0

(c)

Low pressure

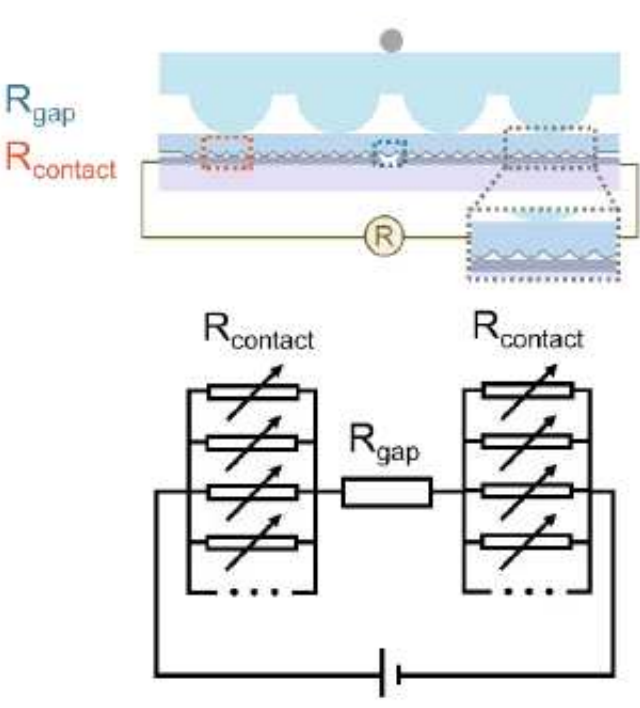

High pressure

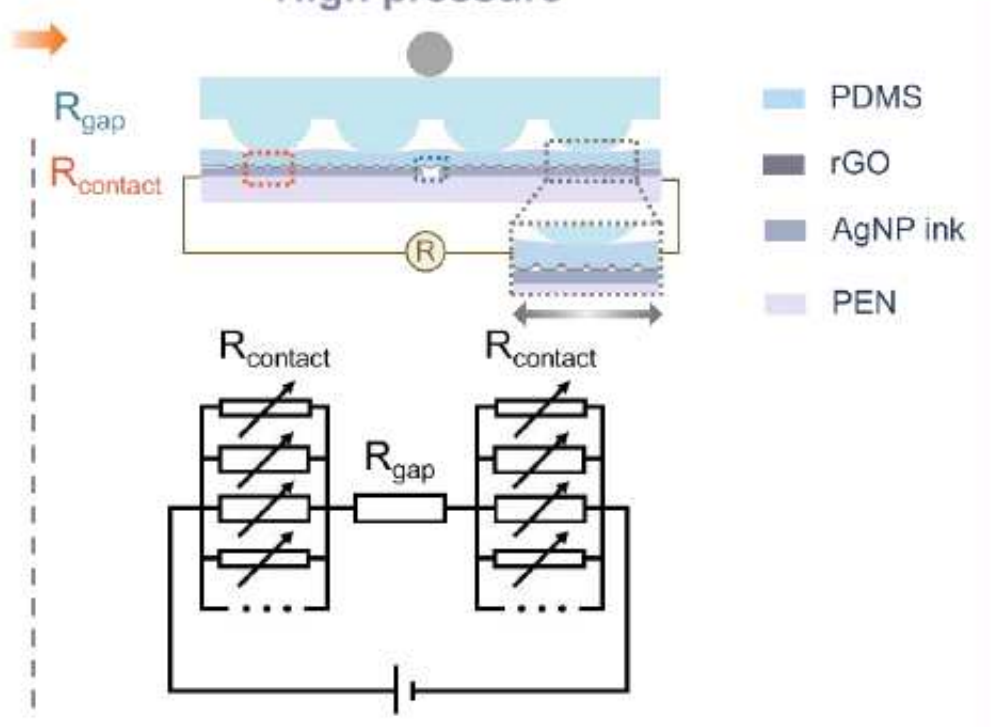

$$
R_{\text {device }}=2 R_{\text {single }}+R_{\text {eqp }}=\frac{2}{n \cdot \frac{1}{R_{\text {contact }}}}+R_{\text {eqp }}
$$

\section{Figure 4}

Working mechanism of the HB pressure sensor. (a) 3D model in the finite element analysis (FEA) software corresponding to the schematic diagram of the pressure sensor in Figure 2a. (b) Simulation results of the pressure distribution and deformation of the microstructure in the secondary structural layer as the prescribed displacement increases. (d) Cross-sectional diagrams of the HB graphene sensor and the corresponding circuit diagrams under low pressure and high pressure. 
(a)

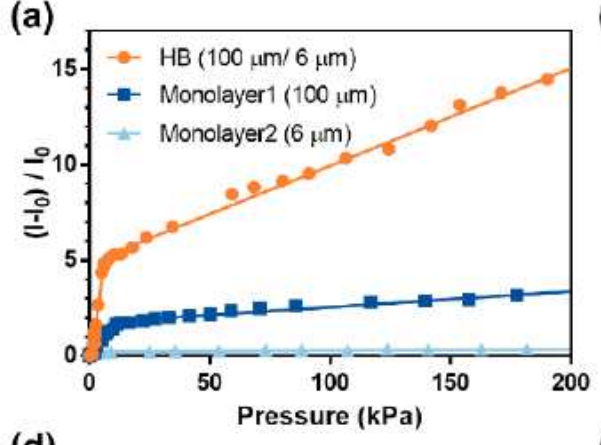

(d)

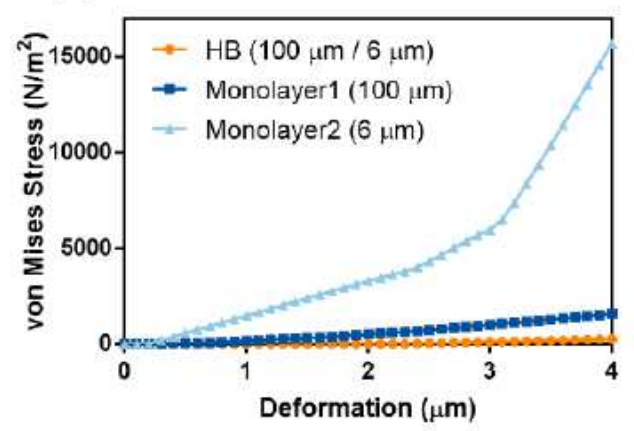

(g)

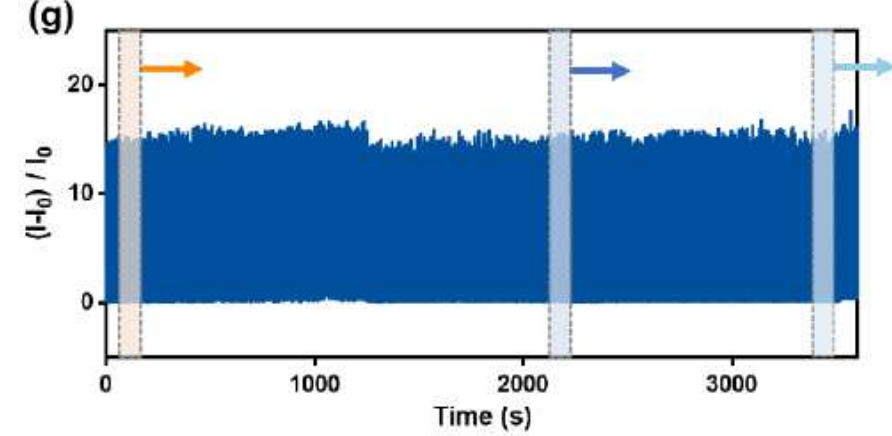

(b)

(e)
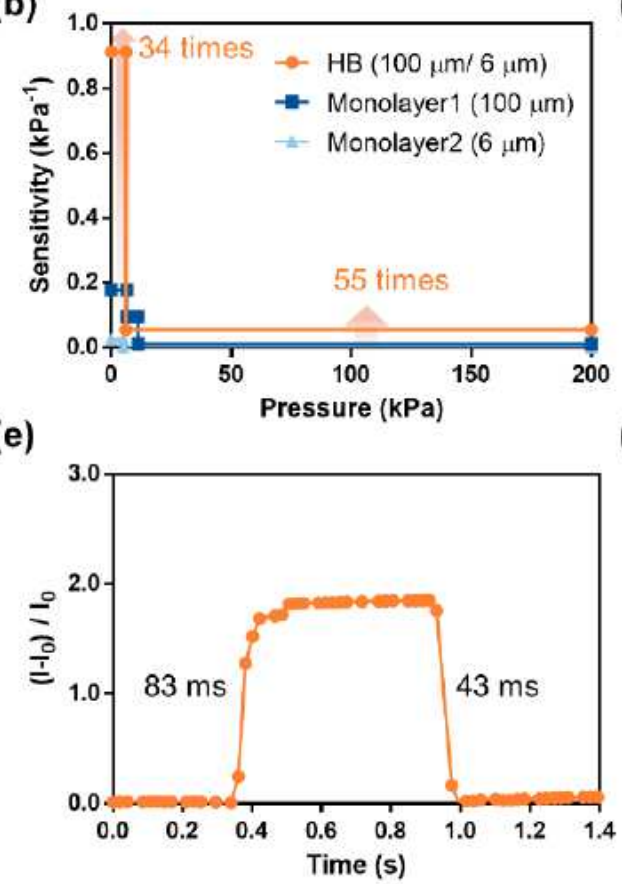

(h)

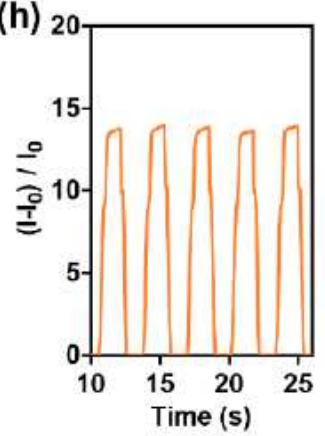

(c)

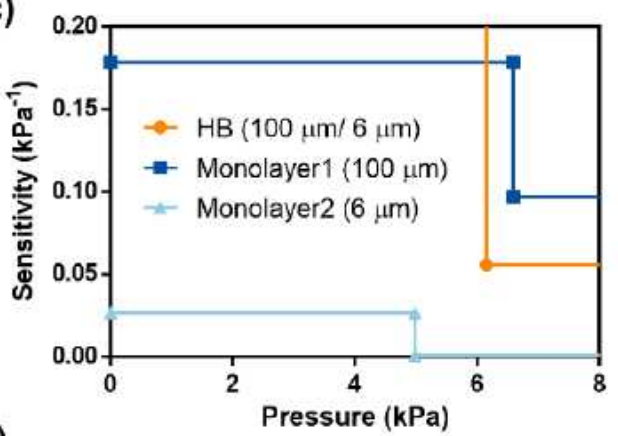

(f)
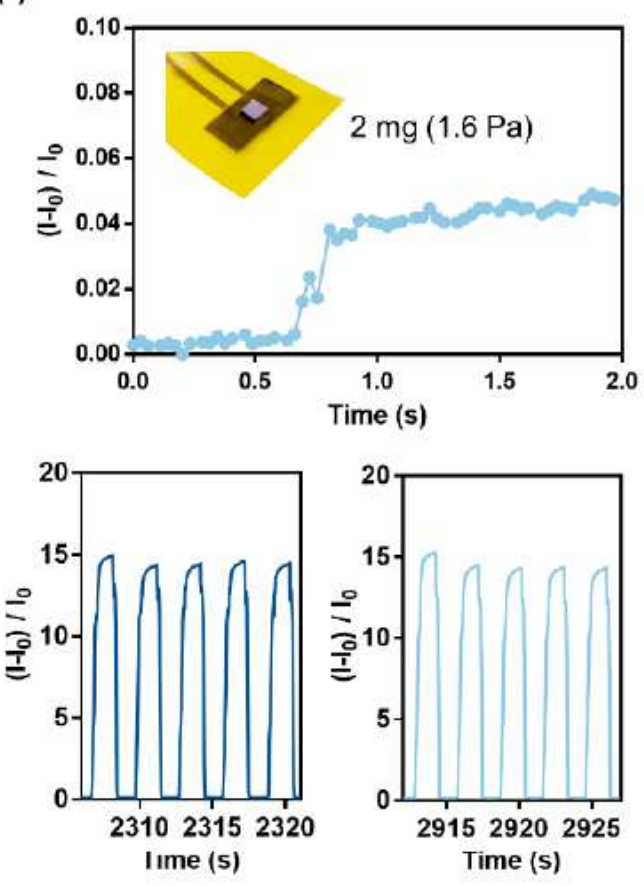

\section{Figure 5}

Electromechanical properties of the HB pressure sensor. (a) Relative current variation versus the pressure on the pressure sensors. (b) Performance comparison and (c) the corresponding magnified graph of the three pressure sensors. (d) von Mises stress versus the prescribed displacement for the three pressure sensors. (e) Response and recovery time of the HB graphene sensor with a load of $1.25 \mathrm{kPa}$. (f) Electrical response of the $\mathrm{HB}$ graphene sensor to the subtle pressure of 1.6 $\mathrm{Pa}$. (g) Relative change in resistance under 1000 cycles of loading pressure of $105 \mathrm{kPa}$. The insets show enlarged views of three time periods. (h) The corresponding magnified graph of the dashed box in ( $g$ ). 
(a)

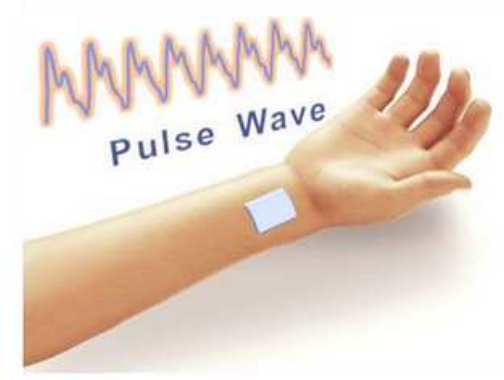

(d)

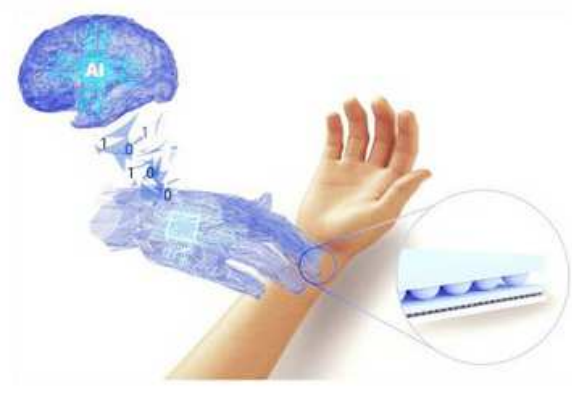

(b)

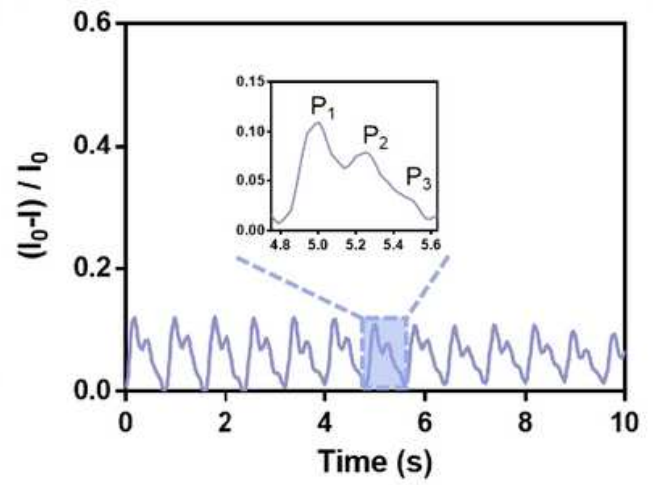

(e)

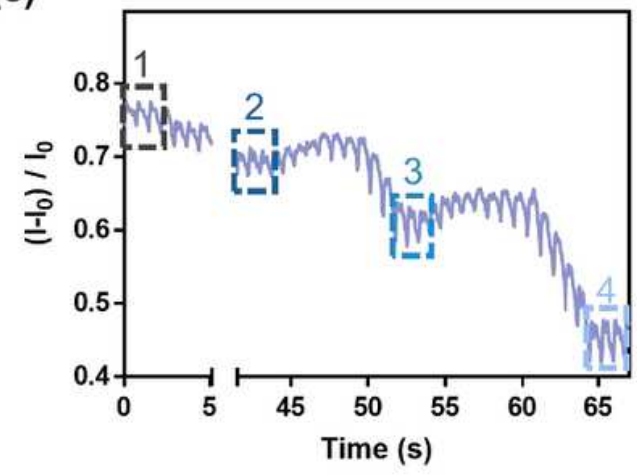

(c)

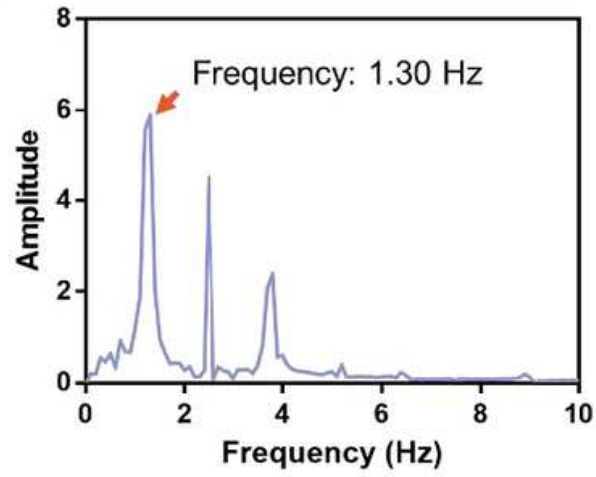

(f)

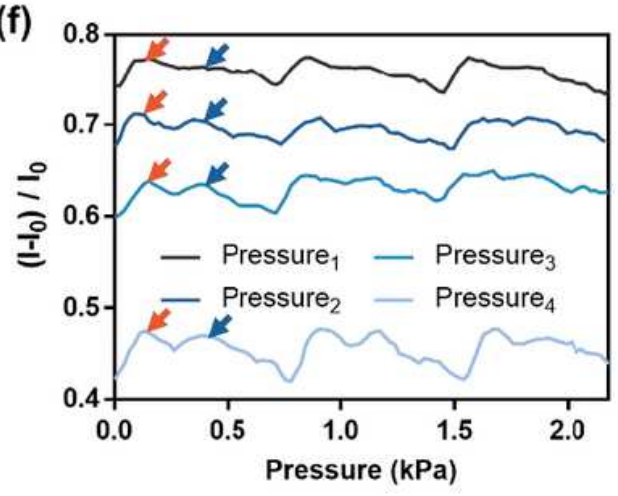

Figure 6

Applications of the HB sensor for pulse monitoring and intelligent pulse diagnosis. (a) Pulse waveform monitoring schematic showing the HB sensor attached to the radial artery of the wrist. (b) Recorded pulse waveforms; inset shows the detailed pulse waves. (c) FFT results of the recorded pulse waveforms. (d) Schematic of intelligent pulse diagnosis. (e) Recorded pulse waveforms under dynamic pulse-taking pressure. (f) Corresponding mapping results among the waves under four different pulse-taking pressures. 
(a)

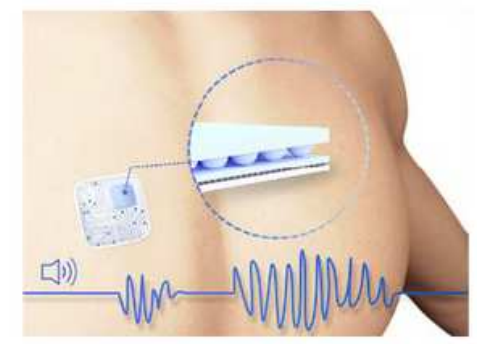

(b)

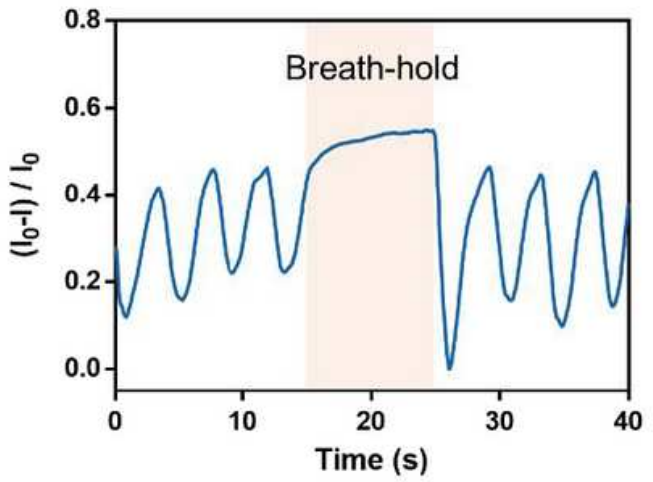

(c)

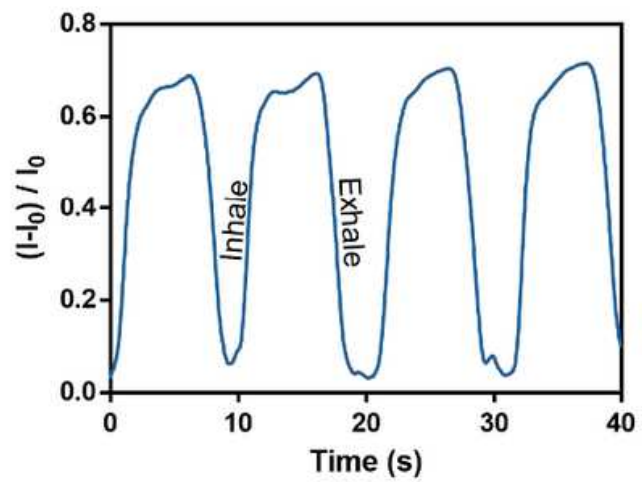

(d)

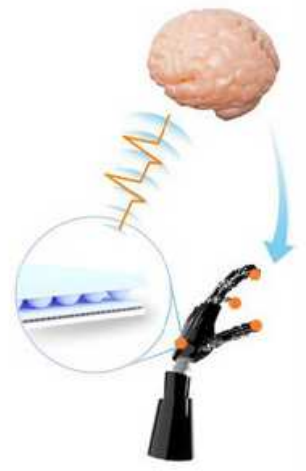

(e)

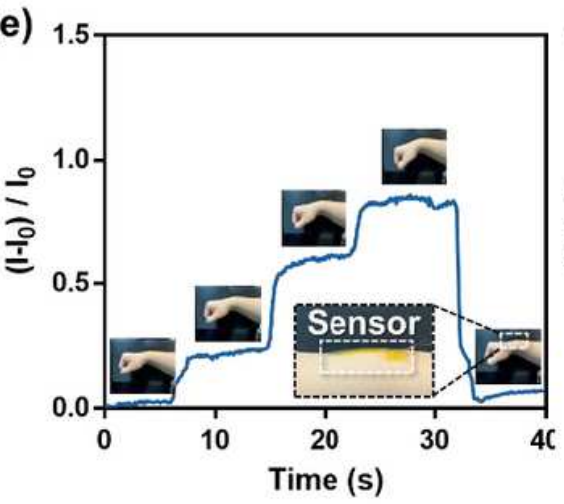

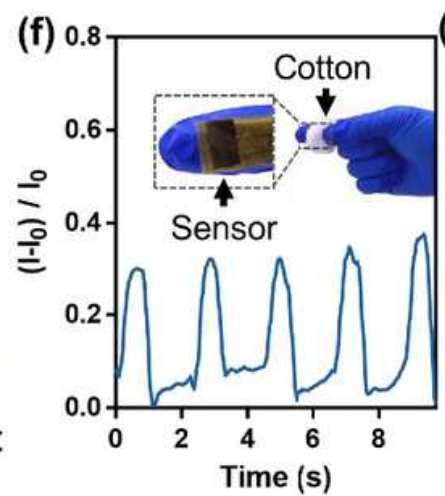

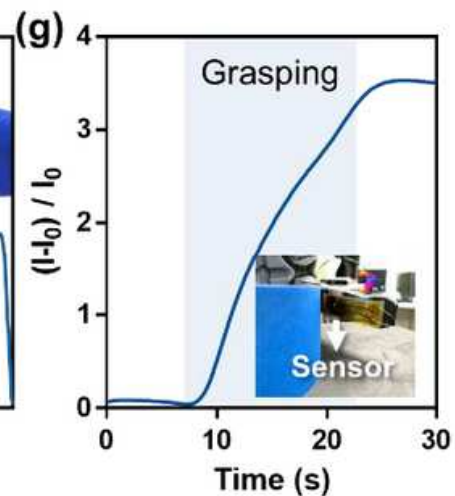

Figure 7

Application of the HB sensor for breath monitoring and electro-tactile feedback. (a) Breath monitoring schematic showing the HB sensor attached to the chest. (b) Monitored normal breathing and simulated apnea signals. (c) Monitored deep breath signals. (d) Application diagram of intelligent prosthesis. (e) Continuous response of the current of the wrist joint under different bending angles. The inset shows the sensor is attached above the wrist using silica gel. (f) Current variation as a function of time as the volunteer grasps a cotton ball. (g) Measured feedback signal from the HB graphene sensor during the capturing process of the manipulator; inset shows an HB sensor attached to the inside of the manipulator.

\section{Supplementary Files}

This is a list of supplementary files associated with this preprint. Click to download.

- MovieS1.mp4 